

\title{
Radiation condition for a non-smooth interface between a dielectric and a metamaterial
}

\author{
Anne-Sophie Bonnet-Ben Dhia, Lucas Chesnel, Xavier Claeys
}

\section{To cite this version:}

Anne-Sophie Bonnet-Ben Dhia, Lucas Chesnel, Xavier Claeys. Radiation condition for a non-smooth interface between a dielectric and a metamaterial. Mathematical Models and Methods in Applied Sciences, 2013. hal-00651008

\section{HAL Id: hal-00651008 \\ https://hal.science/hal-00651008}

Submitted on 12 Dec 2011

HAL is a multi-disciplinary open access archive for the deposit and dissemination of scientific research documents, whether they are published or not. The documents may come from teaching and research institutions in France or abroad, or from public or private research centers.
L'archive ouverte pluridisciplinaire HAL, est destinée au dépôt et à la diffusion de documents scientifiques de niveau recherche, publiés ou non, émanant des établissements d'enseignement et de recherche français ou étrangers, des laboratoires publics ou privés. 


\title{
RADIATION CONDITION FOR A NON-SMOOTH INTERFACE BETWEEN A DIELECTRIC AND A METAMATERIAL
}

\author{
ANNE-SOPHIE BONNET-BEN DHIA \\ Laboratoire POEMS, UMR 7231 CNRS/ENSTA/INRIA, ENSTA ParisTech, \\ 32, boulevard Victor, 75739 Paris Cedex 15, France \\ Anne-Sophie.Bonnet-Bendhia@ensta-paristech.fr \\ LUCAS CHESNEL \\ Laboratoire POEMS, UMR 7231 CNRS/ENSTA/INRIA, ENSTA ParisTech, \\ 32, boulevard Victor, 75739 Paris Cedex 15, France \\ Lucas.Chesnel@ensta-paristech.fr \\ XAVIER CLAEYS \\ Université de Toulouse, ISAE, \\ 10, avenue Edouard-Belin, 31055 Toulouse cedex 4, France \\ Xavier.Claeys@isae.fr
}

December 12, 2011

\begin{abstract}
We study a 2D scalar harmonic wave transmission problem between a classical dielectric and a medium with a real valued negative permittivity/permeability which models an ideal metamaterial. When the interface between the two media has a corner, according to the value of the contrast (ratio) of the physical constants, this non-coercive problem can be ill-posed (not Fredholm) in $H^{1}$. This is due to the degeneration of the two dual singularities which then behave like $r^{ \pm i \eta}=e^{ \pm i \eta \ln r}$ with $\eta \in \mathbb{R}^{*}$. This apparition of propagative singularities is very similar to the apparition of propagative modes in a waveguide for the classical Helmholtz equation with Dirichlet boundary condition, the contrast playing the role of the wavenumber. In this work, we derive for our problem a functional framework by adding to $H^{1}$ one of these propagative singularities. Wellposedness is then obtained by imposing a radiation condition, justified by means of a limiting absorption principle, at the corner between the two media.
\end{abstract}

Keywords: Interface problem; metamaterial; radiation condition; Mellin transform.

\section{Introduction}

The great potential of new artificial materials, called metamaterials, is motivating an intense research in electromagnetics. These metamaterials, which have a complex periodic structure, can be modelized in some frequency range by homogeneous isotropic materials, whose effective dielectric permittivity and magnetic permeability have a negative real part and a small imaginary part ${ }^{30,9,16,25}$. Neglecting losses leads to represent a metamaterial at a given frequency by constants $\varepsilon$ and $\mu$ which are negative real numbers. 
This simple model allows to explain the main phenomena which make the interest of metamaterials. For instance, the unusual negative refraction effect at the interface between a dielectric and a metamaterial is due to the change of sign of $\varepsilon$ and $\mu$. On the other hand, this sign change raises original questions for both the mathematical analysis and the numerical simulation ${ }^{23,26,10}$. Quite general answers have been recently obtained by a variational approach ${ }^{3,2,34,22,1}$. Concerning the time harmonic transmission problem between non-dissipative dielectric and metamaterial, set in a bounded 2D/3D domain, the problem is proved to be of Fredholm type in the classical functional framework if the contrasts (ratios of the values of $\varepsilon$ and $\mu$ across the interface) are outside some intervals, which always contain the value -1 . These intervals reduce to $\{-1\}$ if (and only if) the interface is a regular curve/surface ${ }^{7}$. Up to now, the effect of the corners of the interface, noticed for instance in ${ }^{28,31}$, was not very well understood from a mathematical point of view, and clarifying this question is precisely the goal of this paper.

For that, we consider the simplest case of a static 2D problem, which reduces to the Laplace scalar equation (2.1) with a piecewise constant coefficient $\sigma$ whose sign changes across a polygonal interface. The coefficient $\sigma$ may represent, depending on the physical problem under consideration, $\varepsilon, \mu, 1 / \varepsilon$ or $1 / \mu$. Then $\left(\mathrm{cf}^{1}{ }^{1}\right)$ problem (2.1) is of Fredholm type in $H^{1}(\Omega)$ if and only if the contrast $\kappa_{\sigma}$ defined by $(2.2)$ does not belong to an interval $I$, which is related to the angles of the corners of the interface. Ill-posedness for $\kappa_{\sigma} \in I$ is related to the existence of singular solutions of the equation near the corners, of the form $u=r^{i \eta} \varphi(\theta)$ where $\eta \in \mathbb{R}((r, \theta)$ are local polar coordinates) which do not belong to $H^{1}$. On the other hand, wellposedness in $H^{1}$ can be recovered by taking into account dissipation, adding to $\sigma$ a small imaginary part $\gamma$. A natural question is then the following: is there a functional setting which ensures the well-posedness of the problem for $\kappa_{\sigma} \in I$ (at least Fredholm properties) and such that the solution is the limit of the previous ones when the coefficient of dissipation $\gamma$ tends to 0 .

We answer this question in the present paper, and for the sake of simplicity, we consider a particular geometry (cf. figure 2.1) which allows explicit calculations of the singular exponents (at the singular point $O$ ). Extension to a general polygonal interface does not raise additional difficulties. But difficult questions remain unsolved in several more general configurations $\left(L^{\infty}\right.$ coefficient $\sigma, 3 \mathrm{D}$ geometries ...).

Let us underline that there is a strong analogy between the problem under consideration in the present article and scattering problems in unbounded domains. Indeed, for the latters, the so called limiting absorption principle links the $H^{1}$ solution of the dissipative problem with the outgoing (not $H^{1}$ ) solution of the non dissipative one. The analogy appears clearly by using the Euler coordinates $(z, \theta)$ with $z=\ln r$ near the corner: then the neighborhood of the corner becomes a semiinfinite strip and the singular solutions of the form $r^{i \eta} \varphi(\theta)$ correspond to propagative modes $e^{i \eta z} \varphi(\theta)$ in the strip. And the idea is simply to obtain the appropriate functional setting for our corner problem by selecting the "outgoing" solution in 
the strip. The theoretical justification of this choice is more intricate and requires adapting Mellin techniques in Kondrat'ev weighted Sobolev spaces (introduced in ${ }^{11}$ ) to a non-elliptic equation (because of the change of sign of $\sigma$ ). We hope that the analogy with scattering theory will help those readers which are not familiar with Mellin techniques in understanding the paper.

This unusual phenomenon of "propagative singularity" has been already mentioned in other physical domains and referred to as a "black-hole" phenomenon. It appears for instance in elastodynamics (or in water waves) if the geometrical domain exhibits cuspidal singularities $5,6,19,20,21$. In practice, black-holes are hard to observe since an ideal cuspidal singularity cannot be manufactured and experiments are done with the help of damping materials ${ }^{14}$. To our knowledge, the question whether black-holes due to corners of metamaterials can be observed in practice is an open question.

The outline of the paper is the following. The definition of the problem and the notations are introduced in Section 2. We recall in Section 3 how the so-called $T$ coercivity technique ${ }^{1}$ allows to prove that the problem is well-posed in $H^{1}(\Omega)$ when the contrast $\kappa_{\sigma} \notin[-1,-1 / 3]$. Section 4 is the main part of the paper and contains all the new results for the case $\kappa_{\sigma} \in[-1,-1 / 3]$. Well-posedness in an appropriate functional setting is established together with a limiting absorption principle. Some technical parts of the proofs are postponed to Section 5, where all the details are given.

\section{Setting of the problem}

We consider an open subset $\Omega \subset \mathbb{R}^{2}$ with polygonal boundary such as represented in Figure 1. We assume that $\Omega$ is partitioned in two sub-domains $\Omega_{1}, \Omega_{2}$ so that $\bar{\Omega}=\bar{\Omega}_{1} \cup \bar{\Omega}_{2}$ with $\Omega_{1} \cap \Omega_{2}=\emptyset$. In addition, we assume that the interface $\Sigma=\bar{\Omega}_{1} \cap \bar{\Omega}_{2}$ is a straight segment that intersects $\partial \Omega$ at only two points $O$ and $O^{\prime}$ that are not located at corners of $\partial \Omega$. In the sequel, we shall often refer to the polar coordinates $(r, \theta)$ centered at $O$, and such that $\theta=0$ or $\pi$ at the boundary in the neighbourhood of $O$.

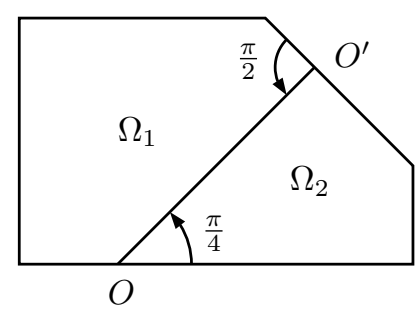

Fig. 1. Geometry of the problem. 
At $O^{\prime}$, we assume that $\Sigma$ is perpendicular to $\partial \Omega$. Finally, we suppose that there exists a ball $\mathrm{B}\left(0, r_{0}\right)$ centered at $O$ such that $\Omega_{2} \cap \mathrm{B}\left(0, r_{0}\right)=\{(r \cos \theta, r \sin \theta) \in$ $\left.\mathbb{R}^{2} \mid 0<r<r_{0}, 0<\theta<\pi / 4\right\}$ and $\Omega_{1} \cap \mathrm{B}\left(0, r_{0}\right)=\left\{(r \cos \theta, r \sin \theta) \in \mathbb{R}^{2} \mid 0<r<\right.$ $\left.r_{0}, \pi / 4<\theta<3 \pi / 4\right\}$. We consider the value $\pi / 4$ for the opening of the cone simply because it will allow explicit calculus in Section 4 . There is no difficulty to adapt the rest of the forthcoming analysis for other values of this angle. The problem that we wish to study in this article writes

$$
-\operatorname{div}(\sigma \nabla u)=f \quad \text { in } \Omega, \text { and } u=0 \text { on } \partial \Omega .
$$

We shall give more details later concerning the space where $u$ is to be sought and $f$ to be chosen. The function $\sigma$ is supposed to be piecewise constant: $\sigma=\sigma_{j}$ in $\Omega_{j}$, $j=1,2$, where $\sigma_{1}$ and $\sigma_{2}$ are two constants such that $\sigma_{1}>0$ and $\sigma_{2}<0$. Let us underline that, in the sequel, the following contrast parameter will play a key role

$$
\kappa_{\sigma} \stackrel{\text { def }}{=} \frac{\sigma_{2}}{\sigma_{1}} \text {. }
$$

Note that considering a problem of the form $-\operatorname{div}(\sigma \nabla u)+\mu u=f$ in $\Omega$ with $\mu \in \mathrm{L}^{\infty}(\Omega)$ and $u=0$ on $\partial \Omega$ (which covers the case of the time harmonic regime) rather than (2.1) would only induce minor corrections in the analysis we provide in the present article.

Before proceeding further, let us introduce some basic notations. For an open subset $\mathcal{O} \subset \mathbb{R}^{d}$ with $d=1,2$, the product of $\mathrm{L}^{2}(\mathcal{O})$ (respectively $\mathrm{L}^{2}(\mathcal{O})^{2}$ ) will be denoted

$$
(u, v)_{\mathcal{O}} \stackrel{\text { def }}{=} \int_{\mathcal{O}} u(\mathbf{x}) v(\mathbf{x}) d \mathbf{x}, \quad \forall u, v \in \mathrm{L}^{2}(\mathcal{O})\left(\text { resp. } \mathrm{L}^{2}(\mathcal{O})^{2}\right) .
$$

Hence, the standard scalar product in $\mathrm{L}^{2}(\mathcal{O})\left(\right.$ resp. $\left.\mathrm{L}^{2}(\mathcal{O})^{2}\right)$ is $(u, v) \mapsto(u, \bar{v})_{\mathcal{O}}$. We set $\|u\|_{\mathrm{L}^{2}(\mathcal{O})} \stackrel{\text { def }}{=}(u, \bar{u})_{\mathcal{O}}^{1 / 2}$ and $\|u\|_{\mathrm{H}_{0}^{1}(\mathcal{O})} \stackrel{\text { def }}{=}\|\nabla u\|_{\mathrm{L}^{2}(\mathcal{O})}$. We set $\langle,\rangle_{\mathcal{O}}$ as the duality pairing between $\mathrm{H}^{-1}(\mathcal{O})$ and $\mathrm{H}_{0}^{1}(\mathcal{O})$, and define the norm

$$
\|u\|_{\mathrm{H}^{-1}(\mathcal{O})} \stackrel{\text { def }}{=} \sup _{v \in \mathrm{H}_{0}^{1}(\mathcal{O})} \frac{\left|\langle u, v\rangle_{\mathcal{O}}\right|}{\|v\|_{\mathrm{H}_{0}^{1}(\mathcal{O})}}, \quad \forall u \in \mathrm{H}^{-1}(\mathcal{O})
$$

These notations allow to introduce a continuous linear operator $A: \mathrm{H}_{0}^{1}(\Omega) \rightarrow$ $\mathrm{H}^{-1}(\Omega)$ defined by

$$
\langle A u, v\rangle_{\Omega} \stackrel{\text { def }}{=}(\sigma \nabla u, \nabla v)_{\Omega}, \quad \forall u, v \in \mathrm{H}_{0}^{1}(\Omega) .
$$

In the sequel, on several occasions, we shall rely on Fredholm theory using the following definition.

Definition 2.1. Let $\mathrm{X}$ and $\mathrm{Y}$ be two Banach spaces, and let $L: \mathrm{X} \rightarrow \mathrm{Y}$ be a continuous linear map. The operator $L$ is said to be of Fredholm type if and only if the following two conditions are fulfilled

i) $\operatorname{dim}(\operatorname{ker} L)<\infty$; 
ii) $\operatorname{dim}(\operatorname{coker} L)<\infty$ where coker $L \stackrel{\text { def }}{=}(\mathrm{Y} /$ range $L)$.

Besides, the index of a Fredholm operator $L$ is defined by $\operatorname{ind}(L)=\operatorname{dim}(\operatorname{ker} L)-$ $\operatorname{dim}(\operatorname{coker} L)$.

\section{The case of a non critical contrast}

Let us examine whether (2.1) is well-posed in a standard Sobolev setting. In the case $\kappa_{\sigma} \in \mathbb{C} \backslash(-\infty, 0]$, it is classically a consequence of Lax-Milgram theorem that there exists a unique $u \in \mathrm{H}_{0}^{1}(\Omega)$ solution to (2.1) for all $f \in \mathrm{H}^{-1}(\Omega)$. Hence, in the remaining of the present section, we focus on the case $\kappa_{\sigma} \in(-\infty, 0)$.

\subsection{Non critical real negative contrast}

In the case $\kappa_{\sigma} \in(-\infty, 0)$, the analysis presented in ${ }^{3,1}$ shows that Problem (2.1) fits the Fredholm framework in classical Sobolev spaces whenever the contrast parameter $\kappa_{\sigma}$ remains outside some critical interval.

Proposition 3.1. For $\kappa_{\sigma} \in(-\infty, 0) \backslash[-1,-1 / 3]$, the operator $A$ defined by (2.3) is a Fredholm operator and $\operatorname{ind}(A)=0$.

We do not provide a detailed proof of Proposition 3.1, but briefly sketch it. For further details, we refer the reader to ${ }^{1}$. First of all, observe that $A$ is self-adjoint. As a consequence, according to Lemma 6.1, it suffices to show that there exists $C>0$ such that

$$
\|u\|_{\mathrm{H}_{0}^{1}(\Omega)} \leq C\left(\|A u\|_{\mathrm{H}^{-1}(\Omega)}+\|u\|_{\mathrm{L}^{2}(\Omega)}\right), \quad \forall u \in \mathrm{H}_{0}^{1}(\Omega) .
$$

We make use of a partition of unity, so that establishing (3.1) boils down to obtaining a similar estimate local to each element of the partition, which is done by applying the technique of $T$-coercivity. The partition of unity is constructed in such a manner that it suffices to derive an estimate of the form (3.1) in two different model geometries. We present in detail how to treat each of these model cases.

The first one is a symmetric domain with straight interface (see Figure 2). This case is supposed to model Problem (2.1) when localized around any point of the interface except $O$.

Lemma 3.1. For some $a, b>0$, consider the domain $\omega=(-a, a) \times(-b, b)$, and denote $\omega_{1}=(-a, a) \times(0, b)$ and $\omega_{2}=(-a, a) \times(-b, 0)$. Set $\sigma=\sigma_{j}$ in $\omega_{j}$ with $\sigma_{1}>0$ and $\sigma_{2}<0$. Under the assumption that

$$
\kappa_{\sigma}=\sigma_{2} / \sigma_{1} \neq-1
$$

the operator $\mathcal{A}: \mathrm{H}_{0}^{1}(\omega) \rightarrow \mathrm{H}^{-1}(\omega)$ defined by $\langle\mathcal{A} u, v\rangle_{\omega}=(\sigma \nabla u, \nabla v)_{\omega}$ is an isomorphism mapping $\mathrm{H}_{0}^{1}(\omega)$ onto $\mathrm{H}^{-1}(\omega)$. 



Fig. 2. Geometry of the first model problem.

Proof. As announced previously, we are going to use the technique of $T$-coercivity. Define the map $R_{1}: \mathrm{H}^{1}\left(\omega_{1}\right) \rightarrow \mathrm{H}^{1}\left(\omega_{2}\right)$ by $\left(R_{1} v\right)(x, y)=v(x,-y)$, and the map $R_{2}: \mathrm{H}^{1}\left(\omega_{2}\right) \rightarrow \mathrm{H}^{1}\left(\omega_{1}\right)$ by $\left(R_{2} v\right)(x, y)=v(x,-y)$. Consider the two operators $T_{1}, T_{2}$ mapping $\mathrm{H}_{0}^{1}(\omega)$ into $\mathrm{H}_{0}^{1}(\omega)$ and defined by

$$
T_{1} u=\left\{\begin{array}{ll}
u_{1} & \text { in } \omega_{1} \\
-u_{2}+2 R_{1} u_{1} & \text { in } \omega_{2}
\end{array} \quad \text { and } \quad T_{2} u= \begin{cases}-u_{1}+2 R_{2} u_{2} & \text { in } \omega_{1} \\
u_{2} & \text { in } \omega_{2}\end{cases}\right.
$$

where $u_{j}=\left.u\right|_{\omega_{j}}, j=1,2$. Observe that for each $j=1,2$, the operator $T_{j}$ is an isomorphism satisfying $T_{j} \cdot T_{j}=\mathrm{Id}$. Let us bound $\left(\sigma \nabla u, \nabla\left(T_{1} \bar{u}\right)\right)_{\omega}$ from below for any $u \in \mathrm{H}_{0}^{1}(\omega)$. Applying Young's inequality with a parameter $\delta>0$, and taking into account the definition of $T_{1}$, we obtain

$$
\begin{gathered}
\Re e\left\{\left(\sigma \nabla u, \nabla\left(T_{1} \bar{u}\right)\right)_{\omega}\right\}=\left(\sigma_{1} \nabla u_{1}, \nabla \bar{u}_{1}\right)_{\omega_{1}}+\left(\left|\sigma_{2}\right| \nabla u_{2}, \nabla \bar{u}_{2}\right)_{\omega_{2}} \\
-2 \Re e\left\{\left(\left|\sigma_{2}\right| \nabla u_{2}, \nabla\left(R_{1} \bar{u}_{1}\right)\right)_{\omega_{2}}\right\} \\
\geq\left(\sigma_{1} \nabla u_{1}, \nabla \bar{u}_{1}\right)_{\omega_{1}}+\left(\left|\sigma_{2}\right| \nabla u_{2}, \nabla \bar{u}_{2}\right)_{\omega_{2}} \\
\quad-\delta\left(\left|\sigma_{2}\right| \nabla u_{2}, \nabla \bar{u}_{2}\right)_{\omega_{2}}-\frac{1}{\delta}\left(\left|\sigma_{2}\right| \nabla\left(R_{1} u_{1}\right), \nabla\left(R_{1} \bar{u}_{1}\right)\right)_{\omega_{2}} \\
\geq\left(\left(\sigma_{1}-\|\left. R_{1}\right|^{2} \frac{\left|\sigma_{2}\right|}{\delta}\right) \nabla u_{1}, \nabla \bar{u}_{1}\right)_{\omega_{1}}+\left(\left|\sigma_{2}\right|(1-\delta) \nabla u_{2}, \nabla \bar{u}_{2}\right)_{\omega_{2}} .
\end{gathered}
$$

The inequality above holds for any $\delta>0$. Exchanging the role of $\omega_{1}$ and $\omega_{2}$, one may apply the same calculus with $T_{2}$ instead of $T_{1}$. This yields, for all $\delta>0$,

$$
\begin{aligned}
\Re e\left\{\left(\sigma \nabla u, \nabla\left(T_{2} \bar{u}\right)\right)_{\omega}\right\} \geq & \left(\sigma_{1}(1-\delta) \nabla u_{1}, \nabla \bar{u}_{1}\right)_{\omega_{1}} \\
& +\left(\left(\left|\sigma_{2}\right|-\left\|R_{2}\right\|^{2} \sigma_{1} / \delta\right) \nabla u_{2}, \nabla \bar{u}_{2}\right)_{\omega_{2}} .
\end{aligned}
$$

Clearly $\left\|R_{1}\right\|=\left\|R_{2}\right\|=1$. Considering the first (resp. second) above inequality if $\sigma_{1}>\left|\sigma_{2}\right|$ (resp. $\sigma_{1}<\left|\sigma_{2}\right|$ ) and taking $\delta$ such that $\left|\sigma_{2}\right| / \sigma_{1}<\delta<1$ (resp. $\delta$ such that $\sigma_{1} /\left|\sigma_{2}\right|<\delta<1$ ), one concludes that there exists an isomorphism $T: \mathrm{H}_{0}^{1}(\omega) \rightarrow \mathrm{H}_{0}^{1}(\omega)$ and a constant $C>0$ such that

$$
\Re e\left\{(\sigma \nabla u, \nabla(T \bar{u}))_{\omega}\right\} \geq C\|u\|_{\mathrm{H}_{0}^{1}(\omega)}^{2}, \quad \forall u \in \mathrm{H}_{0}^{1}(\omega) .
$$


Hence, according to Lax-Milgram theorem applied to the sesquilinear form $(u, v) \mapsto$ $(\sigma \nabla u, \nabla(T \bar{v}))_{\omega}$, for any $f \in \mathrm{H}^{-1}(\omega)$ there exists a unique $u \in \mathrm{H}_{0}^{1}(\omega)$ such that $\langle\mathcal{A} u, T \bar{v}\rangle_{\omega}=(\sigma \nabla u, \nabla(T \bar{v}))_{\omega}=\langle f, T \bar{v}\rangle_{\omega}$ for all $v \in \mathrm{H}_{0}^{1}(\omega)$. Since $T$ is an isomorphism, this proves that $\mathcal{A}$ is an isomorphism as well.

The second toy geometry that we consider is supposed to model Problem (2.1) when localized around $O$.

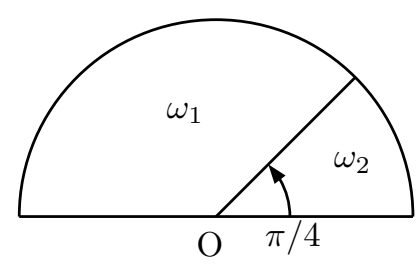

Fig. 3. Geometry of the second model problem.

Lemma 3.2. Let $\omega$ be the open upper half unit disc defined by $\bar{\omega}=\bar{\omega}_{1} \cup \bar{\omega}_{2}$ with $\omega_{1}=\{(r \cos \theta, r \sin \theta) \mid 0<r<1, \pi / 4<\theta<\pi\}$ and $\omega_{2}=\{(r \cos \theta, r \sin \theta) \mid 0<$ $r<1, \quad 0<\theta<\pi / 4\}$. Set $\sigma=\sigma_{j}$ in $\omega_{j}$ with $\sigma_{1}>0$ and $\sigma_{2}<0$. Under the assumption that

$$
\kappa_{\sigma}=\sigma_{2} / \sigma_{1} \notin[-1,-1 / 3],
$$

the operator $\mathcal{A}: \mathrm{H}_{0}^{1}(\omega) \rightarrow \mathrm{H}^{-1}(\omega)$ defined by $\langle\mathcal{A} u, v\rangle_{\omega}=(\sigma \nabla u, \nabla v)_{\omega}$ is an isomorphism mapping $\mathrm{H}_{0}^{1}(\omega)$ onto $\mathrm{H}^{-1}(\omega)$.

Proof. Let $T_{1}, T_{2}: \mathrm{H}_{0}^{1}(\omega) \rightarrow \mathrm{H}_{0}^{1}(\omega)$ be defined by (3.2) where the operators $R_{1}, R_{2}$ are now different, being defined as follows

$$
\left(R_{1} u_{1}\right)(r, \theta)=u_{1}(r, \pi-3 \theta) \quad \text { and } \quad\left(R_{2} u_{2}\right)(r, \theta)=\left\{\begin{array}{cc}
u_{2}(r, \pi / 2-\theta) & \text { if } \theta \leq \pi / 2 \\
0 & \text { if } \theta \geq \pi / 2
\end{array} .\right.
$$

Checking that $\left\|R_{2}\right\|=1$ is straightforward. In order to compute $\left\|R_{1}\right\|$, consider the change of variables $(r, \theta)=(\rho,(\pi-\Theta) / 3)$. We have

$$
\begin{aligned}
\left\|\nabla\left(R_{1} u_{1}\right)\right\|_{\mathrm{L}^{2}\left(\omega_{2}\right)}^{2} & =\int_{\omega_{2}}\left|\frac{\partial\left(R_{1} u_{1}\right)}{\partial r}\right|^{2}+\frac{1}{r^{2}}\left|\frac{\partial\left(R_{1} u_{1}\right)}{\partial \theta}\right|^{2} r d r d \theta \\
& =3 \int_{\omega_{1}}\left|\frac{\partial u_{1}}{\partial \rho}\right|^{2} \rho d \rho d \Theta+\frac{1}{3} \int_{\omega_{1}} \frac{1}{\rho^{2}}\left|\frac{\partial u_{1}}{\partial \Theta}\right|^{2} \rho d \rho d \Theta \leq 3\left\|\nabla u_{1}\right\|_{\mathrm{L}^{2}\left(\omega_{1}\right)}^{2} .
\end{aligned}
$$

As a consequence, we see that $\left\|R_{1}\right\|^{2} \leq 3$. Now, we can apply once again a calculus similar to (3.3) either with $T_{1}$ or $T_{2}$. This time, the value of the parameter $\delta$ has to be chosen in accordance with $\left\|R_{1}\right\|$ or $\left\|R_{2}\right\|$. This finally leads to the conclusion that, whenever $\sigma_{2} / \sigma_{1} \notin[-1,-1 / 3]$, there exists an isomorphism $T: \mathrm{H}_{0}^{1}(\omega) \rightarrow \mathrm{H}_{0}^{1}(\omega)$ 
such that $(u, v) \mapsto(\sigma \nabla u, \nabla(T \bar{v}))_{\omega}$ is coercive, which concludes the proof in the same manner as for Lemma 3.1.

As we explained before, Lemma 3.1 and 3.2 are the two main building blocks for the proof of Proposition 3.1. Note that this proposition does not allow to conclude that Problem (2.1) is well-posed in the case $\sigma_{2} / \sigma_{1} \notin[-1,-1 / 3]$. Uniqueness of the solution has to be assumed in addition.

Corollary 3.1. Assume that $\kappa_{\sigma} \in(-\infty, 0) \backslash[-1,-1 / 3]$. Assume in addition that the only element of $\mathrm{H}_{0}^{1}(\Omega)$ satisfying $\operatorname{div}(\sigma \nabla u)=0$ in $\mathrm{H}^{-1}(\Omega)$ is $u=0$. In this situation the operator $A$ defined by (2.3) is an isomorphism.

\section{The case of a contrast located in the critical interval}

What can we state about the well-posedness or Fredholmness of Problem (2.1) in the case where $\kappa_{\sigma} \in[-1,-1 / 3]$ ? In ${ }^{1}$, it was proved that, as soon as $\Sigma$ presents a straight section, in the case $\kappa_{\sigma}=\sigma_{2} / \sigma_{1}=-1$, the operator $A$ defined by $(2.3)$ does not have a closed range in $\mathrm{H}^{-1}(\Omega)$, hence is not of Fredholm type. The case $\kappa_{\sigma}=-1$ seems to be a particularly problematic situation whose treatment is beyond the scope of the present article. We discard this case from now on and for the rest of this article. In the sequel, we will assume

$$
\kappa_{\sigma}=\frac{\sigma_{2}}{\sigma_{1}} \in(-1,-1 / 3)
$$

Although we shall not consider the value $\kappa_{\sigma}=-1 / 3$ in the present article, we expect that it may be analyzed by some proper modification of the analysis we present for the cases fitting into (4.1).

In the situation of (4.1), observe that all the steps of the proof of Proposition 3.1 still hold except Lemma 3.2. The corner structure of Problem (2.1) around $O$ is the only ingredient that could prevent Proposition 3.1 to hold.

In order to study Problem (2.1) in the neighbourhood of $O$, we will first come back to the reduced problem introduced in Lemma 3.2, which can be rewritten as a waveguide problem, using Euler's change of variable $z=\ln r$. As mentioned in the introduction, explicit calculus shows that the critical interval is characterized by the existence of propagative modes. A brief review of a classical waveguide problem for the Helmholtz equation, for which we know how to deal with these propagative modes, will help us to determine a well-suited functional framework for our problem. Finally, we will extend these results to the initial problem (2.1).

\subsection{Modal analysis}

Consider the domain $\omega$ represented in Figure 3 and introduce the problem

$$
-\operatorname{div}(\sigma \nabla u)=f \quad \text { in } \omega \quad \text { and } \quad u=0 \text { on } \partial \omega .
$$


where the function $\sigma$ depends only on $\theta$ and is given by $\sigma(\theta)=\sigma_{2}$ if $0<\theta<\pi / 4$, and $\sigma(\theta)=\sigma_{1}$ if $\pi / 4<\theta<\pi$.

Problem (4.2) may be reformulated in a domain with an even simpler geometry. Consider the change of variable $z=\ln r$ and set $\widetilde{u}(z, \theta)=u\left(e^{z}, \theta\right)$ and $\widetilde{f}(z, \theta)=$ $e^{2 z} f\left(e^{z}, \theta\right)$. With these notations, Problem (4.2) is changed into a problem set in $\mathcal{B}=(-\infty, 0) \times(0, \pi)$ that writes

$$
-\left(\sigma \partial_{z}^{2}+\partial_{\theta} \sigma \partial_{\theta}\right) \widetilde{u}=\widetilde{f} \text { in } \mathcal{B} \text { and } \widetilde{u}=0 \text { on } \partial \mathcal{B} .
$$

The first equation above can be rewritten $-\operatorname{div}(\sigma \nabla \widetilde{u})=\widetilde{f}$ in $\mathcal{B}$. Because of the geometry of $\mathcal{B}$, it is natural to compute the solutions of the homogeneous problem with separated variables in the infinite strip $\mathbb{R} \times(0, \pi)$, commonly called the modes. More precisely, we look for functions of the form $\exp (\lambda z) \varphi(\theta)$ which satisfy

$$
\operatorname{div}\left(\sigma \nabla\left(e^{\lambda z} \varphi(\theta)\right)\right)=0 \quad \text { in } \quad \mathcal{B} \text { and } \varphi(0)=\varphi(\pi)=0
$$

for some $\lambda \in \mathbb{C}$ whose value is to be determined, and for some $\varphi \in \mathrm{H}^{1}(0, \pi)$. This is equivalent to finding $\lambda \in \mathbb{C}$ and $\varphi \in \mathrm{H}_{0}^{1}(0, \pi)$ satisfying $\mathscr{L}(\lambda) \varphi=0$ where $\mathscr{L}(\lambda): \mathrm{H}_{0}^{1}(0, \pi) \rightarrow \mathrm{H}^{-1}(0, \pi)$ is the continuous operator defined by

$$
\langle\mathscr{L}(\lambda) \varphi, \psi\rangle_{(0, \pi)}=\int_{0}^{\pi} \sigma \partial_{\theta} \varphi \partial_{\theta} \psi d \theta-\lambda^{2} \int_{0}^{\pi} \sigma \varphi \psi d \theta, \quad \forall \varphi, \psi \in \mathrm{H}_{0}^{1}(0, \pi) .
$$

The corresponding $\lambda$ will be called an eigenvalue of the pencil $\mathscr{L}$, and the set of eigenvalues of $\mathscr{L}$ will be denoted $\Lambda\left(\kappa_{\sigma}\right)$.

Although, as an eigenvalue problem, (4.5) is linear in $\lambda^{2}$, it does not fit any well established framework because of the sign change of $\sigma$ across the interface $\theta=$ $\pi / 4$. One surprising feature of this problem, for example, is that purely complex eigenvalues may appear, as we shall see later.

Let us first examine whether 0 may be an eigenvalue of $\mathscr{L}$. This implies that $\partial_{\theta}^{2} \varphi=0$ separately in $(0, \pi / 4)$ and in $(\pi / 4, \pi)$, so that there exist two constants $\alpha, \beta \in \mathbb{C}$ such that $\varphi(\theta)=\alpha \theta$ in $(0, \pi / 4)$, and $\varphi(\theta)=\beta(\theta-\pi)$ in $(\pi / 4, \pi)$. The transmission conditions at $\theta=\pi / 4$ are satisfied if both $\alpha \pi / 4=-3 \beta \pi / 4$ and $\alpha \sigma_{2}=\beta \sigma_{1}$. This is only possible if $\sigma_{2} / \sigma_{1}=-1 / 3$. In other words,

$$
0 \text { is an eigenvalue of Problem (4.4) } \Longleftrightarrow \quad \kappa_{\sigma}=\frac{\sigma_{2}}{\sigma_{1}}=-\frac{1}{3} .
$$

Now, let us study the modes associated with eigenvalues different from 0 . Consider $\lambda \neq 0$ such that there exists $\varphi \in \mathrm{H}_{0}^{1}(0, \pi)$ satisfying $\mathscr{L}(\lambda) \varphi=0$. This implies that $\partial_{\theta}^{2} \varphi+\lambda^{2} \varphi=0$ separately in $(0, \pi / 4)$ and in $(\pi / 4, \pi)$. Since $\varphi(0)=\varphi(\pi)=0$, there must exist constants $\alpha, \beta \in \mathbb{C}$ such that $\varphi(\theta)=\alpha \sin (\lambda \theta)$ for $\theta \in(0, \pi / 4)$ and $\varphi(\theta)=\beta \sin (\lambda(\theta-\pi))$ for $\theta \in(\pi / 4, \pi)$. Imposing the transmission conditions at $\pi / 4$ leads to solving the following equations

$$
\alpha \sin \left(\frac{\lambda \pi}{4}\right)+\beta \sin \left(\frac{3 \lambda \pi}{4}\right)=0 \quad \text { and } \quad \alpha \sigma_{2} \cos \left(\frac{\lambda \pi}{4}\right)-\beta \sigma_{1} \cos \left(\frac{3 \lambda \pi}{4}\right)=0 .
$$


The second equation required to divide by $\lambda$ which is a valid operation as $\lambda \neq 0$. The linear equations above admit a non trivial solution if and only if its determinant vanishes, which leads to the dispersion relation

$$
\sigma_{1} \sin \left(\frac{\lambda \pi}{4}\right) \cos \left(\frac{3 \lambda \pi}{4}\right)+\sigma_{2} \cos \left(\frac{\lambda \pi}{4}\right) \sin \left(\frac{3 \lambda \pi}{4}\right)=0 .
$$

To simplify this relation, use the formula $2\left(a_{1} b_{1}+a_{2} b_{2}\right)=\left(a_{1}+a_{2}\right)\left(b_{1}+b_{2}\right)+$ $\left(a_{1}-a_{2}\right)\left(b_{1}-b_{2}\right)$ where $a_{j}=\sigma_{j}, j=1,2, b_{1}=\sin (\lambda \pi / 4) \cos (3 \lambda \pi / 4)$ and $b_{2}=$ $\cos (\lambda \pi / 4) \sin (3 \lambda \pi / 4)$. By means of elementary trigonometric formulas, this yields

$$
2\left(\sigma_{1}+\sigma_{2}\right) \sin \left(\frac{\lambda \pi}{2}\right) \cos \left(\frac{\lambda \pi}{2}\right)=\left(\sigma_{1}-\sigma_{2}\right) \sin \left(\frac{\lambda \pi}{2}\right) .
$$

This relation is satisfied when $\sin (\lambda \pi / 2)=0$, i.e. for the case $\lambda \in 2 \mathbb{Z} \backslash\{0\}$ (we have assumed that $\lambda \neq 0$ ). In the case where $\lambda \notin 2 \mathbb{Z}$, then we can further simplify and obtain

$$
\cos \left(\frac{\lambda \pi}{2}\right)=\rho\left(\kappa_{\sigma}\right) \quad \text { with } \quad \rho\left(\kappa_{\sigma}\right) \stackrel{\text { def }}{=} \frac{1}{2} \frac{1-\kappa_{\sigma}}{1+\kappa_{\sigma}} .
$$

Note that, for $\kappa_{\sigma} \in \mathbb{R}$, we have $\rho\left(\kappa_{\sigma}\right) \in[-1,+1]$ if and only if $\kappa_{\sigma} \in \mathbb{R} \backslash(-1,-1 / 3]$. In (4.7) it also appears clearly that the value $\kappa_{\sigma}=-1$ would raise difficulties. The previous discussion on eigenvalues leads to the conclusion that the set of eigenvalues of $\mathscr{L}$ is given by the following expression (recall that $z \mapsto-i \ln \left(z+i \sqrt{1-z^{2}}\right)$ is the natural extension to the complex plane of the function arccos )

$$
\begin{aligned}
& \Lambda\left(\kappa_{\sigma}\right)=(2 \mathbb{Z} \backslash\{0\}) \cup\left\{i \eta_{\sigma}+4 \mathbb{Z}\right\} \cup\left\{-i \eta_{\sigma}+4 \mathbb{Z}\right\} \\
& \text { with } \eta_{\sigma}=-\frac{2}{\pi} \ln \left(\rho\left(\kappa_{\sigma}\right)+i \sqrt{1-\rho\left(\kappa_{\sigma}\right)^{2}}\right) .
\end{aligned}
$$

Formula (4.8) shows that, for $\kappa_{\sigma} \in(-1,-1 / 3)$, we have $\Im m\left\{\eta_{\sigma}\right\}=0$, see Figure 4 below and ${ }^{8,4,27}$, and $\Re e\left\{\eta_{\sigma}\right\}>0$, so that $\pm i \eta_{\sigma}$ are two purely imaginary eigenvalues. The associated modes take the form

$$
u_{\mathrm{p}}^{ \pm}(z, \theta)=\varphi_{\mathrm{p}}(\theta) e^{ \pm i \eta_{\sigma} z} \text { with } \varphi_{\mathrm{p}}(\theta)= \begin{cases}\frac{\sinh \left(\eta_{\sigma} \theta\right)}{\sinh \left(\eta_{\sigma} \pi / 4\right)} & \text { on }[0, \pi / 4] \\ \frac{\sinh \left(\eta_{\sigma}(\pi-\theta)\right)}{\sinh \left(\eta_{\sigma} 3 \pi / 4\right)} & \text { on }[\pi / 4, \pi] .\end{cases}
$$

By analogy with classical waveguide problems (see the next subsection), these modes will be called propagative modes.

In the case where $\kappa_{\sigma} \in(-\infty, 0) \backslash[-1,-1 / 3]$, all eigenvalues have their real part different from 0, see Figure 4, so that all modes are evanescent.

To sum up, let us emphasize that the critical interval $(-1,-1 / 3)$ is characterized by the existence of propagative modes. 


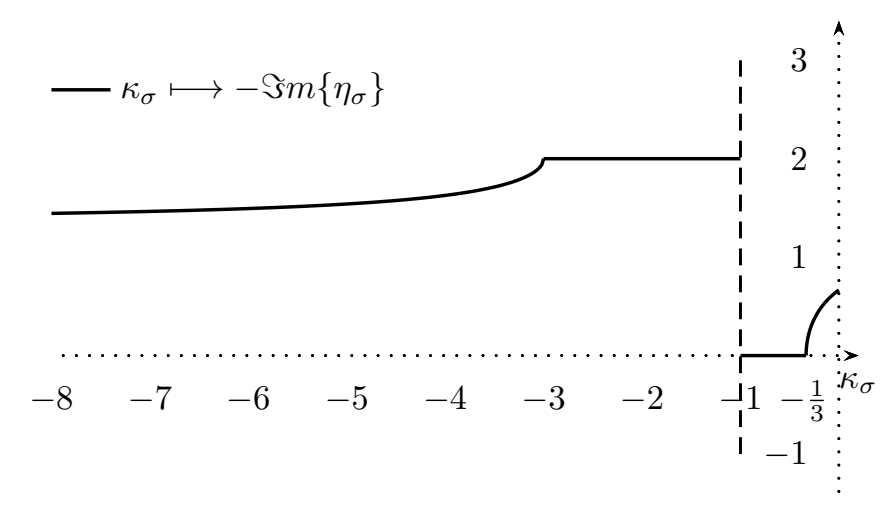

Fig. 4. $\kappa_{\sigma} \mapsto-\Im m\left\{\eta_{\sigma}\right\}$ for $\kappa_{\sigma} \in(-8,0)$.

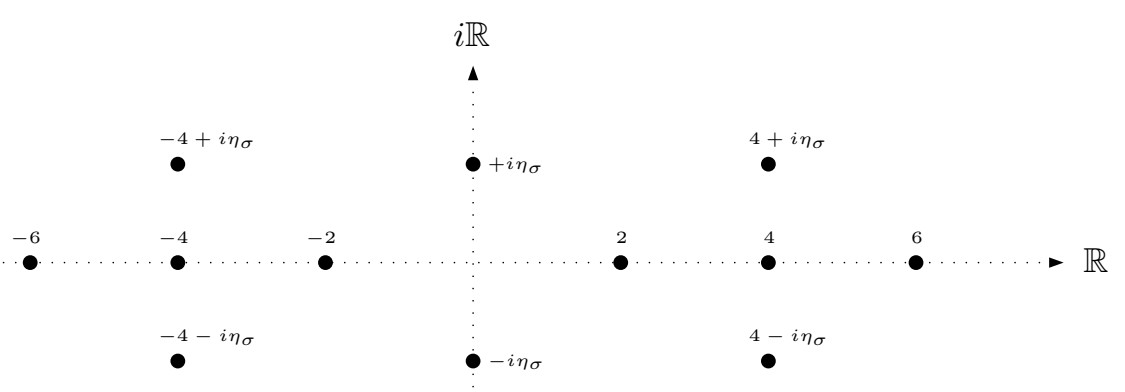

Fig. 5. Location of the eigenvalues for $\kappa_{\sigma}=-\frac{1}{2} \in(-1,-1 / 3)$.

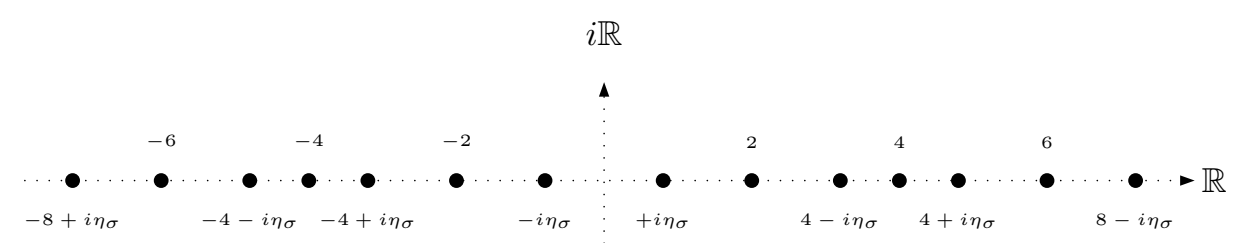

Fig. 6. Location of the eigenvalues for $\kappa_{\sigma}=-\frac{1}{4}$.

Remark 4.1. The modal analysis that we have just presented still holds in the case $\kappa_{\sigma} \in \mathbb{C}^{*} \backslash[-1,-1 / 3]$. In particular the eigenvalues may still be computed by 
means of Formula (4.8). The determination of the logarithm that we consider is defined by $\ln \left(r e^{i \alpha}\right)=\ln r+i \alpha$ for $r \in(0,+\infty), \alpha \in(-\pi,+\pi]$, and the square root is defined by $\sqrt{r e^{i \alpha}}=\sqrt{r} e^{i \alpha / 2}$ for $r \in(0,+\infty), \alpha \in(-\pi,+\pi]$.

Remark 4.2. When $\kappa_{\sigma} \in(-\infty, 0) \backslash[-1,-1 / 3]$, we have seen that all modes are evanescent, and it would be possible to prove well posedness of $(4.3)$ in $\mathrm{H}_{0}^{1}(\mathcal{B})$ using the techniques presented in the next sections, in accordance with Lemma 3.2.

\subsection{A brief review of a standard waveguide problem}

In order to obtain some insight on problem (4.3), let us take a look at a more classical waveguide problem: find $v \in \mathrm{H}_{\mathrm{loc}}^{1}(\mathcal{B})$ such that

$$
\left\{\begin{array}{l}
\Delta v+k^{2} v=0 \text { in } \mathcal{B}, \\
v(z, 0)=v(z, \pi)=0 \quad \forall z \leq 0, \\
v(0, \theta)=g(\theta),
\end{array}\right.
$$

where $k \in \mathbb{R}_{+} \backslash \mathbb{N}$ and $g \in \mathrm{H}_{00}^{1 / 2}(0, \pi)$. Again, separation of variables is a natural approach and computing the modes leads to consider the following eigenvalue problem

$$
\varphi \in \mathrm{H}_{0}^{1}(0, \pi), \quad \int_{0}^{\pi} \partial_{\theta} \varphi \partial_{\theta} \psi-k^{2} \varphi \psi d \theta=\lambda^{2} \int_{0}^{\pi} \varphi \psi d \theta, \quad \forall \psi \in \mathrm{H}_{0}^{1}(0, \pi) .
$$

One straightforwardly finds that the modes form a family $\left(\exp \left( \pm \lambda_{n} z\right) \varphi_{n}(\theta)\right)_{n \in \mathbb{N}^{*}}$ given by

$$
\varphi_{n}(\theta)=\sin (n \theta) \quad \text { and } \quad \lambda_{n}= \begin{cases}-i \sqrt{k^{2}-n^{2}} & \text { if } n \leq k, \\ \sqrt{n^{2}-k^{2}} & \text { if } n \geq k .\end{cases}
$$

For $k<1$, all $\lambda_{n}$ are positive real numbers so that all modes are evanescent (as for the problem studied in the previous section for a contrast outside the critical interval). Propagative modes appear for $k>1$. In particular, for $k \in(1,2)$ there exist exactly two propagative modes $\sin \theta e^{ \pm i \sqrt{k^{2}-1} z}$, propagating in opposite directions. We will focus on this situation which is analogous to what happens for a contrast lying inside the critical interval.

First notice that uniqueness does not hold for problem (4.10). Indeed, $\sin \theta \sin \left(\sqrt{k^{2}-1} z\right)$ is a solution for $g=0$. Classically, well-posedness is recovered by imposing a radiation condition, to select the "outgoing" solution. Since (4.11) is a self-adjoint eigenvalue problem, the family $(\sqrt{2 / \pi} \sin (n \theta))_{n>0}$ is a Hilbert basis of $\mathrm{L}^{2}(0, \pi)$. Using the representation of $g$ on this basis $g(\theta)=\sum_{n=1}^{\infty} g_{n} \sin (n \theta)$, the outgoing solution of (4.10) writes (assuming a time dependence in $\exp \left(-i \omega_{0} t\right)$ with a pulsation $\left.\omega_{0}>0\right)$

$$
v(z, \theta)=g_{1} \sin \theta e^{-i \sqrt{k^{2}-1} z}+v_{e}(z, \theta),
$$


where the evanescent component reads $v_{e}(z, \theta)=\sum_{n=2}^{\infty} g_{n} \sin (n \theta) e^{\sqrt{n^{2}-k^{2}} z}$.

Remark 4.3. A classical way to characterize the outgoing solution is to use the limiting absorption principle. Consider Problem (4.10) where $k$ has been replaced by $k_{\epsilon}=k+i \epsilon$. Such problem has a unique solution $v_{\epsilon}$ in $\mathrm{H}^{1}(\mathcal{B})$. The outgoing solution to (4.10) is then defined as the limit $v$ of $v_{\epsilon}$ in $\mathrm{H}_{\mathrm{loc}}^{1}(\mathcal{B})$ as $\epsilon \rightarrow 0$.

\subsection{Well-posedness within an adapted functional setting}

Now we come back to Problem (4.3). We want to find a functional setting in which it would be well posed. For this, a natural idea consists in trying to adapt the approach recalled in $\S 4.2$. Unfortunately, because of the sign change of $\sigma$ across the interface $\theta=\pi / 4$, the eigenvalue problem associated with (4.5) is not self-adjoint and we are not able to justify some basis property for the corresponding eigenvectors. Nevertheless, using (4.12) as a guideline, we will look for a solution which is the sum of one propagative mode and an evanescent part. The weighted Sobolev spaces that we will introduce now are well-suited to measure the exponentially decaying behaviour at infinity of this evanescent component.

Let $\mathscr{C}_{0}^{\infty}(\mathcal{B})$ refer to the set of $\mathscr{C}^{\infty}$-functions whose support is compactly embedded in $\mathcal{B}$. For $k \geq 0$, let $\mathrm{W}_{\beta}^{k}(\mathcal{B})$ refer to the closure of $\mathscr{C}_{0}^{\infty}(\mathcal{B})$ with respect to the norm defined by

$$
\|v\|_{\mathrm{W}_{\beta}^{k}(\mathcal{B})} \stackrel{\text { def }}{=}\left(\sum_{\alpha+\gamma \leq k}\left\|e^{\beta z} \partial_{z}^{\alpha} \partial_{\theta}^{\gamma} v\right\|_{\mathrm{L}^{2}(\mathcal{B})}^{2}\right)^{1 / 2} .
$$

This norm is equivalent to the norm $\left\|e^{\beta z} v\right\|_{\mathrm{H}^{k}(\mathcal{B})}$ and, we have $\mathrm{W}_{0}^{1}(\mathcal{B})=\mathrm{H}_{0}^{1}(\mathcal{B})$ and $\mathrm{W}_{0}^{0}(\mathcal{B})=\mathrm{L}^{2}(\mathcal{B})$. Besides, if $\beta>0$, we have $\mathrm{W}_{-\beta}^{1}(\mathcal{B}) \subset \mathrm{H}_{0}^{1}(\mathcal{B}) \subset \mathrm{W}_{\beta}^{1}(\mathcal{B})$. To gain insight about these weighted spaces, take a function of the form $v(z, \theta)=$ $\varphi(\theta) \exp (\lambda z) \zeta(z)$ where $\varphi \in \mathscr{C}^{\infty}(0, \pi) \cap \mathrm{H}_{0}^{1}(0, \pi), \lambda \in \mathbb{C}, \zeta(z) \in \mathscr{C}^{\infty}(\mathbb{R}), \zeta(0)=0$ and $\zeta(z)=1$ for $z \leq-1$. Then $v \in \mathrm{W}_{\beta}^{1}(\mathcal{B})$ if and only if $\Re e\{\lambda\}>-\beta$.

We shall denote $\mathrm{W}_{\beta}^{1}(\mathcal{B})^{*}$ the topological dual to $\mathrm{W}_{\beta}^{1}(\mathcal{B})$, and $\langle,\rangle_{\mathcal{B}}$ the duality pairing between $\mathrm{W}_{\beta}^{1}(\mathcal{B})^{*}$ and $\mathrm{W}_{\beta}^{1}(\mathcal{B})$. We equip this space with the norm

$$
\|v\|_{\mathrm{W}_{\beta}^{1}(\mathcal{B})^{*}}=\sup _{u \in \mathrm{W}_{\beta}^{1}(\mathcal{B})} \frac{\left|\langle v, u\rangle_{\mathcal{B}}\right|}{\|u\|_{\mathrm{W}_{\beta}^{1}(\mathcal{B})}}, \quad \forall v \in \mathrm{W}_{\beta}^{1}(\mathcal{B})^{*} .
$$

Observe for example that, for $\beta>0$, we have $\mathrm{W}_{\beta}^{1}(\mathcal{B}) \subset \mathrm{W}_{-\beta}^{1}(\mathcal{B})^{*}$ in the sense that any $u \in \mathrm{W}_{\beta}^{1}(\mathcal{B})$ induces the linear form $v \mapsto(\nabla u, \nabla v)_{\mathcal{B}}$ that is continuous for the norm of $\mathrm{W}_{-\beta}^{1}(\mathcal{B})$. For any $\beta \in \mathbb{R}$, let us denote $L_{\beta}: \mathrm{W}_{\beta}^{1}(\mathcal{B}) \rightarrow \mathrm{W}_{-\beta}^{1}(\mathcal{B})^{*}$ the continuous operator defined by

$$
\left\langle L_{\beta} u, v\right\rangle_{\mathcal{B}}=(\sigma \nabla u, \nabla v)_{\mathcal{B}}, \quad \forall(u, v) \in \mathrm{W}_{\beta}^{1}(\mathcal{B}) \times \mathrm{W}_{-\beta}^{1}(\mathcal{B}) .
$$

For $\kappa_{\sigma} \neq-1$, the following result provides a simple criterion for determining whether the operator $L_{\beta}$ is of Fredholm type. 
Theorem 4.1. Assume that $\kappa_{\sigma}=\sigma_{2} / \sigma_{1} \neq-1$. The operator $L_{\beta}: \mathrm{W}_{\beta}^{1}(\mathcal{B}) \rightarrow$ $\mathrm{W}_{-\beta}^{1}(\mathcal{B})^{*}$ is of Fredholm type if and only if no eigenvalue of $\mathscr{L}(\lambda)$ belongs

$$
\ell_{\beta} \stackrel{\text { def }}{=}\{\lambda \in \mathbb{C} \mid \Re e\{\lambda\}=\beta\} .
$$

The proof relies on arguments very similar to the theory presented in chapter 56 of ${ }^{12}$, and is detailed in Section 5 of the present article. Theorem 4.1 shows in particular that, when $\kappa_{\sigma} \in(-1,-1 / 3), L_{\beta}$ is Fredholm for $\beta \in(-2,2) \backslash\{0\}$. Now, we try to obtain sharper information about $\operatorname{dim}\left(\operatorname{ker} L_{\beta}\right)$.

Lemma 4.1. Let $\kappa_{\sigma} \in(-1,-1 / 3)$ and consider $\beta \in(0,2)$. The operator $L_{-\beta}$ : $\mathrm{W}_{-\beta}^{1}(\mathcal{B}) \rightarrow \mathrm{W}_{\beta}^{1}(\mathcal{B})^{*}$ is one-to-one, and there exists a constant $C>0$ such that

$$
\|v\|_{\mathrm{W}_{-\beta}^{1}(\mathcal{B})} \leq C\left\|L_{-\beta} v\right\|_{\mathrm{W}_{\beta}^{1}(\mathcal{B})^{*}}, \quad \forall v \in \mathrm{W}_{-\beta}^{1}(\mathcal{B}) .
$$

This result will be proved in Section 5. Let $L_{\beta}^{*}$ refer to the adjoint to the operator $L_{\beta}$. Notice that $L_{\beta}^{*}=L_{-\beta}$. Indeed, if $\Lambda\left(\kappa_{\sigma}\right) \cap \ell_{\beta}=\emptyset$, we have $\left\langle L_{\beta}^{*} u, v\right\rangle_{\mathcal{B}}=\left\langle L_{\beta} v, u\right\rangle_{\mathcal{B}}=$ $(\sigma \nabla v, \nabla u)_{\mathcal{B}}=(\sigma \nabla u, \nabla v)_{\mathcal{B}}=\left\langle L_{-\beta} u, v\right\rangle_{\mathcal{B}}$ for $(u, v) \in \mathrm{W}_{-\beta}^{1}(\mathcal{B}) \times \mathrm{W}_{\beta}^{1}(\mathcal{B})$. Moreover, $\operatorname{dim}(\operatorname{coker} L)=\operatorname{dim}\left(\operatorname{ker} L^{*}\right)$ whenever $L: \mathrm{X} \rightarrow \mathrm{Y}$ is a continuous operator with closed range between two Banach spaces $X$ and $Y$, see Theorem 2.13 in ${ }^{17}$. Since $L_{\beta}$ has closed range when $\Lambda\left(\kappa_{\sigma}\right) \cap \ell_{\beta}=\emptyset$, according to Lemma 4.1, we have

$$
\operatorname{dim}\left(\operatorname{coker} L_{\beta}\right)=\operatorname{dim}\left(\operatorname{ker} L_{-\beta}\right)=0 \text { for } \beta \in(0,2) .
$$

To briefly summarize, for $0<\beta<2, L_{-\beta}$ is one-to-one and $L_{\beta}$ is onto. Besides $L_{\beta}$ is not one-to-one, and hence $L_{-\beta}$ is not onto: it is possible to provide an explicit expression of some non trivial element of $\operatorname{ker} L_{\beta}$. Define

$$
s(z, \theta)=-\sin \left(\eta_{\sigma} z\right) \varphi_{\mathrm{p}}(\theta)=\frac{1}{2 i}\left(u_{\mathrm{p}}^{-}(z, \theta)-u_{\mathrm{p}}^{+}(z, \theta)\right) .
$$

where $\varphi_{\mathrm{p}}, u_{\mathrm{p}}^{ \pm}$were defined in Formula (4.9). Then, $s \in \mathrm{W}_{\beta}^{1}(\mathcal{B})$ (notice that $s(0, \theta)=$ $0 \forall \theta \in(0, \pi))$ and $L_{\beta}(s)=0$.

There is some hierarchy between $L_{-\beta}$ and $L_{\beta}$ as we are in the following situation

$$
\begin{array}{cc}
L_{-\beta}: \mathrm{W}_{-\beta}^{1}(\mathcal{B}) & \rightarrow \mathrm{W}_{\beta}^{1}(\mathcal{B})^{*} \\
\cap & \cap \\
L_{\beta}: \mathrm{W}_{\beta}^{1}(\mathcal{B}) & \rightarrow \mathrm{W}_{-\beta}^{1}(\mathcal{B})^{*}
\end{array}
$$

This discussion leads to the intuition that an optimal functional setting leading to a well-posed formulation for Problem (4.3) may consist in a space containing $\mathrm{W}_{-\beta}^{1}(\mathcal{B})$ and included in $\mathrm{W}_{\beta}^{1}(\mathcal{B})$.

Consider a cut-off function $\zeta \in \mathscr{C}^{\infty}(\mathbb{R})$ such that $\zeta(z)=1$ for $z<-2$ and $\zeta(z)=0$ for $z>-1$, and define $s^{ \pm}(z, \theta)=\zeta(z) u_{\mathrm{p}}^{ \pm}(z, \theta)$ where $u_{\mathrm{p}}^{ \pm}$was defined by (4.9). The form $v \mapsto\left(\sigma \nabla s^{ \pm}, \nabla v\right)_{\mathcal{B}}$ is well-defined on $\mathrm{W}_{-\beta}^{1}(\mathcal{B})$. Although $s^{ \pm} \in$ $\mathrm{W}_{\beta}^{1}(\mathcal{B}) \backslash \mathrm{W}_{-\beta}^{1}(\mathcal{B})$, we will extend this linear form to $\mathrm{W}_{\beta}^{1}(\mathcal{B})$, and actually to $\mathrm{W}_{\gamma}^{1}(\mathcal{B})$ for any $\gamma \in \mathbb{R}$. Let us present this extension process for $s^{+}$(one proceeds in the same way for $\left.s^{-}\right)$. Applying Green's formula yields

$$
\left(\sigma \nabla s^{+}, \nabla v\right)_{\mathcal{B}}=\left(\sigma u_{\mathrm{p}}^{+} \nabla \zeta, \nabla v\right)_{\mathcal{B}}-\left(\sigma \nabla u_{\mathrm{p}}^{+}, v \nabla \zeta\right)_{\mathcal{B}}, \quad \forall v \in \mathscr{C}_{0}^{\infty}(\mathcal{B})
$$


since $\operatorname{div}\left(\sigma \nabla u_{\mathrm{p}}^{+}\right)=0$ in $\mathcal{B}$ by construction. As $\operatorname{supp}(\nabla \zeta)$ is bounded, there clearly exists $C>0$ such that $\left|\left(\sigma \nabla s^{+}, \nabla v\right)_{\mathcal{B}}\right| \leq C\|v\|_{\mathrm{W}_{\beta}^{1}(\mathcal{B})}$, for all $v$ in $\mathscr{C}_{0}^{\infty}(\mathcal{B})$. Therefore, according to the density of $\mathscr{C}_{0}^{\infty}(\mathcal{B})$, the linear form $v \mapsto\left(\sigma \nabla s^{ \pm}, \nabla v\right)_{\mathcal{B}}$ can be continuously extended to $\mathrm{W}_{\beta}^{1}(\mathcal{B})$. Now, let us set

$$
\begin{aligned}
& \mathscr{W}_{\beta}^{+}(\mathcal{B})=\operatorname{span}\left\{s^{+}\right\} \oplus \mathrm{W}_{-\beta}^{1}(\mathcal{B}) \quad \text { for } \quad 0<\beta<2 \text { equipped with } \\
& \|v\|_{\mathscr{W}_{\beta}^{+}(\mathcal{B})} \stackrel{\text { def }}{=}\left(|c|^{2}+\left\|v_{-\beta}\right\|_{\mathrm{W}_{-\beta}^{1}(\mathcal{B})}^{2}\right)^{1 / 2} \quad \text { if } \quad v=c s^{+}+v_{-\beta} .
\end{aligned}
$$

The space $\mathscr{W}_{\beta}^{+}(\mathcal{B})$ equipped with this norm is a Banach space that satisfies $\mathrm{W}_{-\beta}^{1}(\mathcal{B}) \subset \mathscr{W}_{\beta}^{+}(\mathcal{B}) \subset \mathrm{W}_{\beta}^{1}(\mathcal{B})$ since $s^{+} \in \mathrm{W}_{\beta}^{1}(\mathcal{B}) \backslash \mathrm{W}_{-\beta}^{1}(\mathcal{B})$. The discussion above shows that there exists a unique linear continuous operator $L_{\beta}^{+}: \mathscr{W}_{\beta}^{+}(\mathcal{B}) \rightarrow \mathrm{W}_{\beta}^{1}(\mathcal{B})^{*}$ satisfying

$$
\left\langle L_{\beta}^{+} u, v\right\rangle_{\mathcal{B}}=(\sigma \nabla u, \nabla v)_{\mathcal{B}}, \quad \forall u \in \mathscr{W}_{\beta}^{+}(\mathcal{B}), \quad \forall v \in \mathscr{C}_{0}^{\infty}(\mathcal{B}) .
$$

We will show that this operator is actually an isomorphism. We first prove an intermediate result providing a more explicit expression of $c$ with respect to $L_{\beta}^{+} u$ when $u=c s^{+}+u_{-\beta} \in \mathscr{W}_{\beta}^{+}(\mathcal{B})$.

Proposition 4.1. Let $\beta \in(0,2)$, and let $s$ be defined by (4.14). For all $u=c s^{+}+$ $u_{-\beta} \in \mathscr{W}_{\beta}^{+}(\mathcal{B})$, where $c \in \mathbb{C}$ and $u_{-\beta} \in \mathrm{W}_{-\beta}^{1}(\mathcal{B})$, the following formula holds

$$
\left\langle L_{\beta}^{+} u, s\right\rangle_{\mathcal{B}}=c \eta_{\sigma} \int_{0}^{\pi} \sigma(\theta) \varphi_{\mathrm{p}}(\theta)^{2} d \theta .
$$

Proof. Since $s \in \mathrm{W}_{\beta}^{1}(\mathcal{B}),\left\langle L_{\beta}^{+} u, s\right\rangle_{\mathcal{B}}$ is well-defined whenever $u \in \mathscr{W}_{\beta}^{+}(\mathcal{B})$. Consider a $\mathscr{C}^{\infty}$ cut-off function $\chi: \mathbb{R} \rightarrow[0,1]$ such that $\chi(z)=1$ for $z>-1$ and $\chi(z)=0$ for $z<-2$. Set $\chi_{\rho}(z)=\chi(z / \rho)$. Observe that $\chi_{\rho} s \in \mathrm{W}_{-\beta}^{1}(\mathcal{B})$ for $\rho>0$. We have

$$
\left\langle L_{\beta}^{+} u, \chi_{\rho} s\right\rangle_{\mathcal{B}}=\left(\sigma \nabla u, \nabla\left(\chi_{\rho} s\right)\right)_{\mathcal{B}}=\left(s \nabla u-u \nabla s, \sigma \nabla \chi_{\rho}\right)_{\mathcal{B}}
$$

because $(\sigma \nabla v, \nabla s)_{\mathcal{B}}=0, \forall v \in \mathrm{W}_{-\beta}^{1}(\mathcal{B})$ (recall that $L_{\beta} s=0$ ). We examine the expression above for $\rho \rightarrow+\infty$. Using the dominated convergence theorem, it is easy to check that $\left\|\chi_{\rho} s-s\right\|_{\mathrm{W}_{\beta}^{1}(\mathcal{B})} \rightarrow 0$ for $\rho \rightarrow+\infty$. Therefore

$$
\lim _{\rho \rightarrow+\infty}\left\langle L_{\beta}^{+} u, \chi_{\rho} s\right\rangle_{\mathcal{B}}=\left\langle L_{\beta}^{+} u, s\right\rangle_{\mathcal{B}}
$$

Now, we examine the behaviour of the right-hand side of (4.17) for $\rho \rightarrow+\infty$. Since $u=c s^{+}+u_{-\beta}$ with $c \in \mathbb{C}$ and $u_{-\beta} \in \mathrm{W}_{-\beta}^{1}(\mathcal{B})$, this right-hand side contains two contributions. The contribution associated with $u_{-\beta}$ satisfies

$$
\begin{gathered}
\left|\left(s \nabla u_{-\beta}-u_{-\beta} \nabla s, \sigma \nabla \chi_{\rho}\right)_{\mathcal{B}}\right| \leq \int_{0}^{\pi} \int_{-2 \rho}^{-\rho}\left|\sigma \partial_{z} \chi_{\rho}\right| \cdot\left|s \nabla u_{-\beta}-u_{-\beta} \nabla s\right| d z d \theta \\
\leq \frac{1}{\rho}\left\|\partial_{z} \chi\right\|_{\mathrm{L}^{\infty}(\mathbb{R})}\left\|u_{-\beta}\right\|_{\mathrm{W}_{-\beta}^{1}(\mathcal{B})}\|s\|_{\mathrm{W}_{\beta}^{1}(\mathcal{B})} \underset{\rho \rightarrow+\infty}{\rightarrow} 0 .
\end{gathered}
$$


To finish the proof, it only remains to examine the contribution associated with $c s^{+}$. Using the fact that $\varphi_{\mathrm{p}}(\theta)$ is real valued, see Formula (4.9), a direct calculus yields

$$
\begin{aligned}
\lim _{\rho \rightarrow+\infty} & \left(s \nabla u-u \nabla s, \sigma \nabla \chi_{\rho}\right)_{\mathcal{B}}=c \lim _{\rho \rightarrow+\infty}\left(s \nabla s^{+}-s^{+} \nabla s, \sigma \nabla \chi_{\rho}\right)_{\mathcal{B}} \\
=c & \lim _{\rho \rightarrow+\infty} \int_{-2 \rho}^{-\rho} \partial_{z} \chi_{\rho} \int_{0}^{\pi} \eta_{\sigma} \sigma \varphi_{\mathrm{p}}(\theta)^{2} d \theta d z=c \eta_{\sigma} \int_{0}^{\pi} \sigma \varphi_{\mathrm{p}}(\theta)^{2} d \theta .
\end{aligned}
$$

To conclude, it suffices to plug (4.18) and (4.19) into (4.17).

Note that the calculus in (4.19) shows in particular that $\left(s \nabla s^{+}-s^{+} \nabla s, \sigma \nabla \chi_{\rho}\right)_{\mathcal{B}}$ is independent of $\rho$ for $\rho$ large enough. Now, we prove that, choosing $\mathscr{W}_{\beta}^{+}(\mathcal{B})$ as a functional setting for (4.3) leads to a well-posed problem.

Theorem 4.2. Consider $\kappa_{\sigma} \in(-1,-1 / 3)$ and $\beta \in(0,2)$. Then the continuous operator $L_{\beta}^{+}: \mathscr{W}_{\beta}^{+}(\mathcal{B}) \rightarrow \mathrm{W}_{\beta}^{1}(\mathcal{B})^{*}$ defined by (4.16) is an isomorphism.

Proof. Take any function $u=c s^{+}+w$ where $w \in \mathrm{W}_{-\beta}^{1}(\mathcal{B})$ such that $\left\langle L_{\beta}^{+} u, v\right\rangle_{\mathcal{B}}=$ $0, \forall v \in \mathscr{C}_{0}^{\infty}(\mathcal{B})$. Proposition 4.1 yields $c \eta_{\sigma} \int_{0}^{\pi} \sigma \varphi(\theta)^{2} d \theta=0$. According to Lemma 6.2 in appendix, this implies that $c=0$ since $\eta_{\sigma} \neq 0$ as $\kappa_{\sigma} \in(-1,-1 / 3)$. Therefore $u \in \mathrm{W}_{-\beta}^{1}(\mathcal{B})$ and it satisfies $(\sigma \nabla u, \nabla v)_{\mathcal{B}}=0, \forall v \in \mathrm{W}_{\beta}^{1}(\mathcal{B})$ which can be rewritten $L_{-\beta} u=0$. According to Lemma 4.1, this implies that $u=0$. Hence, we have obtained that $L_{\beta}^{+}$is one-to-one.

To conclude, it remains to prove that $L_{\beta}^{+}$is onto. Take any $f \in \mathrm{W}_{\beta}^{1}(\mathcal{B})^{*} \subset$ $\mathrm{W}_{-\beta}^{1}(\mathcal{B})^{*}$. Since $L_{\beta}$ is onto, there exists $u_{\beta} \in \mathrm{W}_{\beta}^{1}(\mathcal{B})$ such that $L_{\beta} u_{\beta}=f$. Since $f \in \mathrm{W}_{\beta}^{1}(\mathcal{B})^{*}$, according to Theorem 5.2, the following decomposition holds

$$
u_{\beta}=c^{+} s^{+}+c^{-} s^{-}+u_{-\beta} \quad \text { where } \quad c^{ \pm} \in \mathbb{C} \quad \text { and } \quad u_{-\beta} \in \mathrm{W}_{-\beta}^{1}(\mathcal{B}) .
$$

Now, define $u=u_{\beta}-2 i c^{-} s$ with $s$ defined in (4.14). Since $s \in \operatorname{ker} L_{\beta}$, we have $L_{\beta} u=L_{\beta} u_{\beta}=f$. Besides, we actually have $u \in \mathscr{W}_{\beta}^{+}(\mathcal{B})$, so that $L_{\beta}^{+} u=f$. Hence $L_{\beta}^{+}$is onto.

Notice that we may achieve the same construction as above replacing "+" by "-". Indeed, consider the space $\mathscr{W}_{\beta}^{-}(\mathcal{B})=\operatorname{span}\left\{s^{-}\right\} \oplus \mathrm{W}_{-\beta}^{1}(\mathcal{B})$ for $0<\beta<2$. Changing the previous proof mutatis mutandis we obtain that, for all $f \in \mathrm{W}_{\beta}^{1}(\mathcal{B})^{*}$, there exists a unique $u \in \mathscr{W}_{\beta}^{-}(\mathcal{B})$ such that $(\sigma \nabla u, \nabla v)_{\mathcal{B}}=\langle f, v\rangle_{\mathcal{B}}, \forall v \in \mathscr{C}_{0}^{\infty}(\mathcal{B})$.

Remark 4.4. For $\beta \in(0,2)$, using Theorem 4.2 and Theorem 5.2 , it is straightforward to show that $\operatorname{dim}\left(\operatorname{ker} L_{\beta}\right)=\operatorname{dim}\left(\operatorname{coker} L_{-\beta}\right)=1$. This implies $\operatorname{ind}\left(L_{\beta}\right)=$ $-\operatorname{ind}\left(L_{-\beta}\right)=1$.

\subsection{Limiting absorption principle}

In the preceding paragraph, we introduced two different spaces, namely $\mathscr{W}_{\beta}^{+}(\mathcal{B})$ and $\mathscr{W}_{\beta}^{-}(\mathcal{B})$, and we showed that for any $f \in \mathrm{W}_{\beta}^{1}(\mathcal{B})^{*}$, there exists exactly one 
$u^{+} \in \mathscr{W}_{\beta}^{+}(\mathcal{B})$ and one $u^{-} \in \mathscr{W}_{\beta}^{-}(\mathcal{B})$ such that

$$
\left(\sigma \nabla u^{ \pm}, \nabla v\right)_{\mathcal{B}}=\langle f, v\rangle_{\mathcal{B}}, \forall v \in \mathscr{C}_{0}^{\infty}(\mathcal{B}) .
$$

Any function $\alpha u^{+}+(1-\alpha) u^{-}$, with $\alpha \in \mathbb{C}$, is also a solution to (4.20). Among all such possible solutions, which one is "the" physical solution to Problem (4.3)? Recall that we are considering the case $\kappa_{\sigma}=\sigma_{2} / \sigma_{1} \in(-1,-1 / 3)$. To provide an answer to the previous question, we proceed as mentioned in Remark 4.3, using the limiting absorption principle. Consider a problem analogous to (4.3) with an additional absorption contribution in the coefficient $\sigma$ : for any $f \in \mathrm{W}_{\beta}^{1}(\mathcal{B})^{*}$,

$$
\text { Find } u^{\gamma} \in \mathrm{H}_{0}^{1}(\mathcal{B}) \text { such that } \quad-\operatorname{div}\left(\sigma^{\gamma} \nabla u^{\gamma}\right)=f \quad \text { in } \mathcal{B} .
$$

where $\sigma^{\gamma}(\theta)=\sigma(\theta)(1+i \gamma \operatorname{sign}\{\sigma(\theta)\})$ with $\gamma>0$. Formulation (4.21) has to be understood in the sense of $\mathrm{H}^{-1}(\mathcal{B})$. It is well-posed as the associated sesquilinear form is coercive, and its unique solution is denoted $u^{\gamma} \in \mathrm{H}_{0}^{1}(\mathcal{B})$. The limiting absorption principle consists in stating that $u^{\gamma}$ has to converge toward the physical solution of (4.3) as $\gamma \rightarrow 0_{+}$. In the present situation we will show that $u^{\gamma} \rightarrow u^{+}$in $\mathrm{W}_{\beta}^{1}(\mathcal{B})$ for $\beta \in(0,2)$. This justifies choosing $\mathscr{W}_{\beta}^{+}(\mathcal{B})$ as a proper functional setting for this problem.

Remark 4.5. Here we consider that the choice for the sign "+" in front of " $i \gamma$ " corresponds to an absorbing medium in the time domain. Admittedly such a a choice is far from obvious, but corresponds to the commonly adopted model in the physical literature ${ }^{33,31}$. Let us emphasize that our analysis would also work with the sign "-", leading to choose $u^{-}$as the outgoing solution.

\subsubsection{Modal analysis of the dissipative problem}

As a preliminary step, we formulate observations as regards the modal analysis of Problem (4.21). As was pointed out in Remark 4.1, the eigenvalues of the pencil associated with Problem (4.21) may be computed by means of Formula (4.8). This shows in particular that there exists one eigenvalue $i \eta_{\sigma, \gamma}$ such that $\eta_{\sigma, \gamma} \rightarrow \eta_{\sigma}$ for $\gamma \rightarrow 0_{+}$. By continuity, for a given $\beta \in(0,2)$, there exists $\gamma_{0}>0$ such that, for $\gamma<\gamma_{0},+i \eta_{\sigma, \gamma}$ and $-i \eta_{\sigma, \gamma}$ are the only eigenvalues of Problem (4.21) located in the strip $\{\lambda \in \mathbb{C} \mid-\beta<\Re e\{\lambda\}<+\beta\}$. Let us show that $\Im m\left\{\eta_{\sigma, \gamma}\right\}<0$, at least for $\gamma$ sufficiently small. Denote $\rho_{\gamma}=\rho\left(\kappa_{\sigma}\right)$. Using the expression of $\rho_{\gamma}$ provided by (4.7), one finds

$$
\rho_{\gamma}=\frac{1}{2} \frac{\sigma_{1}(1+i \gamma)-\sigma_{2}(1-i \gamma)}{\sigma_{1}(1+i \gamma)+\sigma_{2}(1-i \gamma)}=\frac{1}{2} \frac{\left(1-\kappa_{\sigma}\right)+i \gamma\left(1+\kappa_{\sigma}\right)}{\left(1+\kappa_{\sigma}\right)+i \gamma\left(1-\kappa_{\sigma}\right)} .
$$

According to (4.7), there holds $\cosh \left(\eta_{\sigma, \gamma} \pi / 2\right)=\rho_{\gamma}$. Denote $\eta_{\sigma, \gamma}^{\prime}=\partial_{\gamma} \eta_{\sigma, \gamma}$. Differentiating with respect to $\gamma$ at 0 , one obtains

$$
\eta_{\sigma, 0}^{\prime} \frac{\pi}{2} \sinh \left(\frac{\eta_{\sigma} \pi}{2}\right)=\frac{2 i \kappa_{\sigma}}{\left(1+\kappa_{\sigma}\right)^{2}} .
$$


Since $\eta_{\sigma} \in \mathbb{R}_{+}^{*}$, this yields $\Im m\left\{\eta_{\sigma, 0}^{\prime}\right\}<0$ and then $\Im m\left\{\eta_{\sigma, \gamma}\right\}<0$ for $\gamma$ small enough. Therefore $i \eta_{\sigma, \gamma}$ is the only eigenvalue of Problem (4.21) located in the strip $\{\lambda \in \mathbb{C} \mid 0<\Re e\{\lambda\}<\beta\}$.

In the sequel, we denote $u_{\mathrm{p}, \gamma}^{+}(z, \theta)\left(\operatorname{resp} . u_{\mathrm{p}, \gamma}^{-}\right)$the eigenmode associated with $i \eta_{\sigma, \gamma}\left(\right.$ resp. $\left.-i \eta_{\sigma, \gamma}\right)$. Here again, the explicit expression of $u_{\mathrm{p}, \gamma}^{ \pm}$is provided by Formula (4.9), replacing $\eta_{\sigma}$ by $\eta_{\sigma, \gamma}$. Define $s_{\gamma}^{ \pm}(\theta, z)=\zeta(z) u_{\mathrm{p}, \gamma}^{ \pm}(z, \theta)$ (where $\zeta$ is the cutoff function that we used for the definition of $s^{ \pm}$). Using dominated convergence theorem, it is easy to check that

$$
\lim _{\gamma \rightarrow 0_{+}}\left\|s_{\gamma}^{-}-s^{-}\right\|_{\mathrm{W}_{\beta}^{1}(\mathcal{B})}=0 \quad \text { and } \quad \lim _{\gamma \rightarrow 0_{+}}\left\|s_{\gamma}^{+}-s^{+}\right\|_{\mathrm{W}_{\beta}^{1}(\mathcal{B})}=0 .
$$

Let us come back to $u^{\gamma} \in \mathrm{H}_{0}^{1}(\mathcal{B})$ the unique solution to Problem (4.21). According to Theorem 5.4.1 in ${ }^{12}$ (which holds for this elliptic problem), for $0<\gamma<\gamma_{0}$, the function $u^{\gamma}$ admits a decomposition of the following form

$$
u^{\gamma}=c^{\gamma} s_{\gamma}^{+}+u_{-\beta}^{\gamma} \quad \text { where } c^{\gamma} \in \mathbb{C}, u_{-\beta}^{\gamma} \in \mathrm{W}_{-\beta}^{1}(\mathcal{B}) .
$$

To prove the convergence of $u^{\gamma}$ to $u^{+} \in \mathscr{W}_{\beta}^{+}(\mathcal{B})$, where $u^{+}$has been defined by (4.20), we will successively prove the convergence of the singularity coefficients $c^{\gamma}$ and the convergence of the evanescent part $u_{-\beta}^{\gamma}$.

\subsubsection{Convergence of the singularity coefficients}

We first prove that $c^{\gamma} \rightarrow c^{+}$for $\gamma \rightarrow 0_{+}$, where $c^{+} \in \mathbb{C}$ is such that $u^{+}=c^{+} s^{+}+u_{-\beta}$ with $u_{-\beta} \in \mathrm{W}_{-\beta}^{1}(\mathcal{B})$. We provide a more explicit expression of $c^{\gamma}$.

Proposition 4.2. Let $\beta \in(0,2)$ and $0<\gamma<\gamma_{0}$. Assume that $\kappa_{\sigma} \in(-1,-1 / 3)$. Define $s_{\gamma}(z, \theta)=-\sin \left(\eta_{\sigma, \gamma} z\right) \varphi_{\mathrm{p}, \gamma}(\theta)$. Consider $u^{\gamma}=c^{\gamma} s_{\gamma}^{+}+u_{-\beta}^{\gamma}$ the unique solution to (4.21). Then we have

$$
\left\langle f, s_{\gamma}\right\rangle_{\mathcal{B}}=c^{\gamma} \eta_{\sigma, \gamma} \int_{0}^{\pi} \sigma^{\gamma} \varphi_{\mathrm{p}, \gamma}(\theta)^{2} d \theta .
$$

The proof is very similar to the proof of Proposition 4.1 (it is also very close to Theorem 5.4.3 in ${ }^{12}$ ), so it is left to the reader. Using (4.22), we see that $\| s_{\gamma}-$ $s \|_{\mathrm{W}_{\beta}^{1}(\mathcal{B})} \rightarrow 0$ for $\gamma \rightarrow 0_{+}$. Besides, we have

$$
\lim _{\gamma \rightarrow 0_{+}} \eta_{\sigma, \gamma} \int_{0}^{\pi} \sigma^{\gamma} \varphi_{\mathrm{p}, \gamma}(\theta)^{2} d \theta=\eta_{\sigma} \int_{0}^{\pi} \sigma \varphi_{\mathrm{p}}(\theta)^{2} d \theta
$$

This shows in particular that, for $\gamma$ small enough $\eta_{\sigma, \gamma} \int_{0}^{\pi} \sigma^{\gamma} \varphi_{\mathrm{p}, \gamma}^{2} d \theta \neq 0$, see Lemma 6.2 (recall that we assumed that $\kappa_{\sigma} \in(-1,-1 / 3)$ ). Applying both Proposition 4.1 and 4.2 yields

$$
\left|c^{+}-c^{\gamma}\right|=\left|\frac{\left\langle f, s_{\gamma}\right\rangle_{\mathcal{B}}}{\eta_{\sigma, \gamma} \int_{0}^{\pi} \sigma^{\gamma} \varphi_{\mathrm{p}, \gamma}^{2} d \theta}-\frac{\langle f, s\rangle_{\mathcal{B}}}{\eta_{\sigma} \int_{0}^{\pi} \sigma \varphi_{\mathrm{p}}^{2} d \theta}\right| \underset{\gamma \rightarrow 0_{+}}{\longrightarrow} 0 .
$$




\subsubsection{Convergence of the evanescent parts}

Now, we prove the convergence of $u_{-\beta}^{\gamma}$ toward $u_{-\beta}$. First of all, recall that both $\operatorname{div}\left(\sigma \nabla s^{+}\right)$and $\operatorname{div}\left(\sigma^{\gamma} \nabla s_{\gamma}^{+}\right)$have compact support since $\operatorname{div}\left(\sigma \nabla u_{\mathrm{p}}^{+}\right)=0$ and $\operatorname{div}\left(\sigma^{\gamma} \nabla u_{\mathrm{p}, \gamma}^{+}\right)=0$ in $\mathcal{B}$ by construction. As $u^{+}=c^{+} s^{+}+u_{-\beta}$ is solution to (4.20) and $u^{\gamma}=c^{\gamma} s_{\gamma}^{+}+u_{-\beta}^{\gamma}$ is solution to $(4.21)$, for any $v \in \mathscr{C}_{0}^{\infty}(\mathcal{B})$, we have

$$
\begin{gathered}
\left|\left(\sigma \nabla\left(u_{-\beta}-u_{-\beta}^{\gamma}\right), \nabla v\right)_{\mathcal{B}}\right| \leq\left|\left(c^{\gamma} \sigma^{\gamma} \nabla s_{\gamma}^{+}-c^{+} \sigma \nabla s^{+}, \nabla v\right)_{\mathcal{B}}\right|+\left|\left(\left(\sigma-\sigma^{\gamma}\right) \nabla u_{-\beta}^{\gamma}, \nabla v\right)_{\mathcal{B}}\right| \\
\leq\left[\left|c^{\gamma}\right|\left\|\operatorname{div}\left(\sigma \nabla s^{+}\right)-\operatorname{div}\left(\sigma^{\gamma} \nabla s_{\gamma}^{+}\right)\right\|_{\mathrm{L}^{2}(\mathcal{B})}+\left|c^{+}-c^{\gamma}\right|\left\|\operatorname{div}\left(\sigma \nabla s^{+}\right)\right\|_{\mathrm{L}^{2}(\mathcal{B})}\right. \\
\left.+\left|\sigma-\sigma^{\gamma}\right|\left\|u_{-\beta}^{\gamma}\right\|_{\mathrm{W}_{-\beta}^{1}(\mathcal{B})}\right]\|v\|_{\mathrm{W}_{\beta}^{1}(\mathcal{B})} .
\end{gathered}
$$

Since $\mathscr{C}_{0}^{\infty}(\mathcal{B})$ is dense in $\mathrm{W}_{\beta}^{1}(\mathcal{B})$, according to the definition of $L_{-\beta}$, the inequality above shows that

$$
\left\|L_{-\beta}\left(u_{-\beta}-u_{-\beta}^{\gamma}\right)\right\|_{\mathrm{W}_{\beta}^{1}(\mathcal{B})^{*}} \leq \epsilon(\gamma)\left(1+\left\|u_{-\beta}^{\gamma}\right\|_{\mathrm{W}_{-\beta}^{1}(\mathcal{B})}\right)
$$

where $\epsilon(\gamma)$ tends to 0 with $\gamma$ (use (4.23) and notice that $\| \operatorname{div}\left(\sigma \nabla s^{+}\right)-$ $\operatorname{div}\left(\sigma^{\gamma} \nabla s_{\gamma}^{+}\right) \|_{\mathrm{L}^{2}(\mathcal{B})} \rightarrow 0$ for $\left.\gamma \rightarrow 0_{+}\right)$. According to lemma 4.1, there exists a constant $C>0$ independent of $\gamma$ such that

$$
\left\|u_{-\beta}-u_{-\beta}^{\gamma}\right\|_{\mathrm{W}_{-\beta}^{1}(\mathcal{B})} \leq C \epsilon(\gamma)\left(1+\left\|u_{-\beta}^{\gamma}\right\|_{\mathrm{W}_{-\beta}^{1}(\mathcal{B})}\right), \quad \forall \gamma \in\left(0, \gamma_{0}\right)
$$

Using triangular inequality, one finally finds

$$
\lim _{\gamma \rightarrow 0_{+}}\left\|u_{-\beta}^{\gamma}-u_{-\beta}\right\|_{\mathrm{W}_{-\beta}^{1}(\mathcal{B})}=0 .
$$

\subsubsection{Final convergence result}

Gathering (4.22), (4.23) and (4.24) finally yields the following theorem:

Theorem 4.3. For $\beta \in(0,2)$, one has

$$
\lim _{\gamma \rightarrow 0_{+}}\left\|u^{\gamma}-u^{+}\right\|_{\mathrm{W}_{\beta}^{1}(\mathcal{B})}=0
$$

where $u^{\gamma}$ is the solution of (4.21) and $u^{+} \in \mathscr{W}_{\beta}^{+}(\mathcal{B})$ the solution of (4.20).

This proves that the solution to a problem of the form (4.21) with a slightly dissipative medium is close to $u^{+}$. Hence $u^{+}$is more relevant than $u^{-}$from a physical point of view.

\subsection{Well-posedness of the initial boundary value problem}

In this paragraph, we want to come to back to the initial problem (2.1), set in the domain $\Omega$ in the case $\kappa_{\sigma} \in(-1,-1 / 3)$. The results that we established in paragraph 4.1 and 4.3 for the problem set in the half-strip $\mathcal{B}$ will help determine an adapted functional setting for this problem. Let us first rewrite these results in the domain $\omega$ 
of Figure 3, using the inverse change of variable $r=\exp z$. We introduce the space $\mathrm{V}_{\beta}^{1}(\omega)$ as the completion of $\mathscr{C}_{0}^{\infty}(\omega)$ with respect to the norm

$$
\|v\|_{\mathrm{V}_{\beta}^{1}(\omega)} \stackrel{\text { def }}{=}\left(\left\|r^{\beta} \nabla v\right\|_{\mathrm{L}^{2}(\omega)}^{2}+\left\|r^{\beta-1} v\right\|_{\mathrm{L}^{2}(\omega)}^{2}\right)^{1 / 2}
$$

It can be checked straightforwardly that $u=u(r, \theta)$ belongs to $\mathrm{V}_{\beta}^{1}(\omega)$ if and only if $\widetilde{u}(z, \theta)=u\left(e^{z}, \theta\right)$ belongs to $\mathrm{W}_{\beta}^{1}(\mathcal{B})$. Let $\zeta: \mathbb{R} \rightarrow[0,1]$ be a $\mathscr{C}^{\infty}$ cut-off function such that $\zeta(r)=1$ for $r<1 / 3$ and $\zeta(r)=0$ for $r>1 / 2$. Mimicing (4.15), we define

$$
\begin{aligned}
& \mathscr{V}_{\beta}^{+}(\omega) \stackrel{\text { def }}{=} \operatorname{span}\left\{r^{i \eta_{\sigma}} \varphi_{p}(\theta) \zeta(r)\right\} \oplus \mathrm{V}_{-\beta}^{1}(\omega) \text { for } 0<\beta<2 \\
& \text { with }\|v\|_{\mathscr{V}_{\beta}^{+}(\omega)} \stackrel{\text { def }}{=}\left(|c|^{2}+\left\|v_{-\beta}\right\|_{\mathrm{V}_{-\beta}^{1}(\omega)}^{2}\right)^{1 / 2} \\
& \text { if } v=c r^{i \eta_{\sigma}} \varphi_{p}(\theta) \zeta(r)+v_{-\beta} .
\end{aligned}
$$

For this functional setting, we also introduce an operator associated with Equation (4.2). In the same manner as for operator $L_{\beta}^{+}$, we define $\mathcal{A}_{\beta}^{+}: \mathscr{V}_{\beta}^{+}(\omega) \rightarrow \mathrm{V}_{\beta}^{1}(\omega)^{*}$ as the unique linear continuous operator satisfying

$$
\left\langle\mathcal{A}_{\beta}^{+} u, v\right\rangle_{\omega}=(\sigma \nabla u, \nabla v)_{\omega}, \quad \forall u \in \mathscr{V}_{\beta}^{+}(\omega), \quad \forall v \in \mathscr{C}_{0}^{\infty}(\omega) .
$$

Adapting the proof of Theorem 4.2 in the coordinates $(r, \theta)$ leads to the following result:

Proposition 4.3. Consider $\kappa_{\sigma} \in(-1,-1 / 3)$ and $\beta \in(0,2)$. Then the continuous operator $\mathcal{A}_{\beta}^{+}: \mathscr{V}_{\beta}^{+}(\omega) \rightarrow \mathrm{V}_{\beta}^{1}(\omega)^{*}$ defined by (4.28) is an isomorphism.

A result similar to Proposition 4.3 with $\omega$ replaced by $\Omega$ does not hold in the general case. However, and this is the main result of this paper, we can prove a slightly weaker version in $\Omega$ (trapped modes can appear). Define $\mathrm{V}_{\beta}^{1}(\Omega)$ as the completion of $\mathscr{C}_{0}^{\infty}(\Omega)$ for the norm \|\|$_{\mathrm{V}_{\beta}^{1}(\Omega)}$ defined by (4.26) where $\omega$ is replaced by $\Omega$. Similarly, define $\mathscr{V}_{\beta}^{+}(\Omega)$ like in (4.27) replacing $\omega$ by $\Omega$. We redefine the functions $s^{ \pm}(r, \theta)=r^{ \pm i \eta_{\sigma}} \varphi_{p}(\theta) \zeta(r)$. Here, of course, the support of the cut-off function $\zeta$ is chosen sufficiently small so that $s^{ \pm}=0$ on $\partial \Omega$.

Proposition 4.4. Consider $\kappa_{\sigma} \in(-1,-1 / 3)$ and $\beta \in(0,2)$. Similar to the definition of $L_{\beta}^{+}$, define $A_{\beta}^{+}: \mathscr{V}_{\beta}^{+}(\Omega) \rightarrow \mathrm{V}_{\beta}^{1}(\Omega)^{*}$ as the unique linear continuous operator satisfying $\left\langle A_{\beta}^{+} u, v\right\rangle_{\Omega}=(\sigma \nabla u, \nabla v)_{\Omega}, \forall u \in \mathscr{V}_{\beta}^{+}(\Omega), \forall v \in \mathscr{C}_{0}^{\infty}(\Omega)$. Then $A_{\beta}^{+}$is a Fredholm operator and $\operatorname{ind}\left(A_{\beta}^{+}\right)=0$.

Proof. We divide the proof in two steps.

Step 1: the operator $A_{\beta}^{+}$IS Of Fredholm type. We proceed as in the proof of Theorem 1.2 of ${ }^{18}$, chapter $4, \S 1$. Let us first introduce some notations. Define the open sets $\omega_{a}=\Omega \cap \mathrm{B}\left(O, d_{a}\right)$ and $\omega_{b}=\Omega \backslash \overline{\mathrm{B}\left(O, d_{b}\right)}$ where $d_{a}$ and $d_{b}$ are chosen so that $0<d_{b}<d_{a}$ and $\left\{(r \cos \theta, r \sin \theta) \in \mathbb{R}^{2} \mid 0<r<d_{a}, 0<\theta<\pi\right\} \subset \Omega$. 
For $\nu=a, b$, let $\zeta_{\nu}$ and $\psi_{\nu}$ be $\mathscr{C}^{\infty}$ functions (with support in $\bar{\Omega}$ ) satisfying the conditions

$$
\left(\Omega \cap \operatorname{supp} \zeta_{\nu}\right) \subset\left(\Omega \cap \operatorname{supp} \psi_{\nu}\right) \subset \omega_{\nu}, \quad \zeta_{\nu} \psi_{\nu}=\zeta_{\nu}, \quad \zeta_{a}+\zeta_{b}=1 \text { in } \Omega .
$$

We also suppose that $\boldsymbol{n} \cdot \nabla \psi_{\nu}=\boldsymbol{n} \cdot \nabla \zeta_{\nu}=0$ on $\Sigma$, where $\boldsymbol{n}$ denotes the unit vector to $\Sigma$ going from $\Omega_{1}$ to $\Omega_{2}$. Introduce $\mathcal{A}_{a}: \mathscr{V}_{\beta}^{+}\left(\omega_{a}\right) \rightarrow \mathrm{V}_{\beta}^{1}\left(\omega_{a}\right)^{*}$ the unique linear continuous operator satisfying $\left\langle\mathcal{A}_{a} u, v\right\rangle_{\omega_{a}}=(\sigma \nabla u, \nabla v)_{\omega_{a}}, \forall u \in \mathscr{V}_{\beta}^{+}\left(\omega_{a}\right)$, $\forall v \in \mathscr{C}_{0}^{\infty}\left(\omega_{a}\right)$. According to Proposition 4.3, $\mathcal{A}_{a}$ is an isomorphism. Introduce also the continuous linear operator $\mathcal{A}_{b}: \mathrm{H}_{0}^{1}\left(\omega_{b}\right) \rightarrow \mathrm{H}^{-1}\left(\omega_{b}\right)$ such that $\left\langle\mathcal{A}_{b} u, v\right\rangle_{\omega_{b}}=$ $(\sigma \nabla u, \nabla v)_{\omega_{b}}+i(u, v)_{\omega_{b}}, \forall u, v \in \mathrm{H}_{0}^{1}\left(\omega_{b}\right)$. With the help of Lemma 3.1, one can prove that $\mathcal{A}_{b}$ is a Fredholm operator of index zero. Since $\mathcal{A}_{b}$ is obviously one-toone (take the imaginary part of $\left\langle\mathcal{A}_{b} u, \bar{u}\right\rangle_{\omega_{b}}$ for $u \in \operatorname{ker} \mathcal{A}_{b}$ ), this implies that $\mathcal{A}_{b}$ is an isomorphism.

Let us prove the following a priori estimate:

$$
\|u\|_{\mathscr{V}_{\beta}^{+}(\Omega)} \leq C\left(\left\|A_{\beta}^{+} u\right\|_{\mathrm{V}_{\beta}^{1}(\Omega)^{*}}+\left\|\psi_{b} u\right\|_{\mathrm{L}^{2}(\Omega)}\right), \quad \forall u \in \mathscr{V}_{\beta}^{+}(\Omega) .
$$

For all $u \in \mathscr{V}_{\beta}^{+}(\Omega)$, there holds

$$
\begin{aligned}
\|u\|_{\mathscr{V}_{\beta}^{+}(\Omega)} & \leq C\left(\left\|\zeta_{a} u\right\|_{\mathscr{V}_{\beta}^{+}\left(\omega_{a}\right)}+\left\|\zeta_{b} u\right\|_{\mathrm{H}_{0}^{1}\left(\omega_{b}\right)}\right) \\
& \leq C\left(\left\|\mathcal{A}_{a}\left(\zeta_{a} u\right)\right\|_{\mathrm{V}_{\beta}^{1}\left(\omega_{a}\right)^{*}}+\left\|\mathcal{A}_{b}\left(\zeta_{b} u\right)\right\|_{\mathrm{H}^{-1}\left(\omega_{b}\right)}\right) \\
& \leq C\left(\left\|\psi_{a} A_{\beta}^{+} u\right\|_{\mathrm{V}_{\beta}^{1}(\Omega)^{*}}+\left\|\psi_{b} A_{\beta}^{+} u\right\|_{\mathrm{V}_{\beta}^{1}(\Omega)^{*}}+\left\|\psi_{b} u\right\|_{\mathrm{L}^{2}(\Omega)}\right) \\
& \leq C\left(\left\|A_{\beta}^{+} u\right\|_{\mathrm{V}_{\beta}^{1}(\Omega)^{*}}+\left\|\psi_{b} u\right\|_{\mathrm{L}^{2}(\Omega)}\right)
\end{aligned}
$$

Since the map $u \mapsto \psi_{b} u$ from $\mathscr{V}_{\beta}^{+}(\Omega)$ to $\mathrm{L}^{2}(\Omega)$ is compact (recall that $\psi_{b}$ vanishes in the neighbourhood of $O$ ), one deduces from Lemma 6.1 that range $A_{\beta}^{+}$is closed and $\operatorname{ker} A_{\beta}^{+}$has finite dimension.

Now, let us build a right regularizer (also called a right parametrix), i.e. an operator $\mathrm{R}$ such that $A_{\beta}^{+} \mathrm{R}-\mathrm{Id}$ is a compact operator of $\mathrm{V}_{\beta}^{1}(\Omega)^{*}$. According to lemma 2.23 in ${ }^{17}$, this will prove that coker $A_{\beta}^{+}$is finite-dimensional. Define the operator

$$
\mathrm{R} \stackrel{\text { def }}{=} \zeta_{a} \mathcal{A}_{a}^{-1} \psi_{a}+\zeta_{b} \mathcal{A}_{b}^{-1} \psi_{b}
$$

For all $f \in \mathrm{V}_{\beta}^{1}(\Omega)^{*}$, one finds

$$
\begin{aligned}
A_{\beta}^{+} \mathrm{R} f & =A_{\beta}^{+} \zeta_{a} \mathcal{A}_{a}^{-1} \psi_{a} f+A_{\beta}^{+} \zeta_{b} \mathcal{A}_{b}^{-1} \psi_{b} f \\
& =\zeta_{a} f+\zeta_{b} f+\left[A_{\beta}^{+}, \zeta_{a}\right] \mathcal{A}_{a}^{-1} \psi_{a} f+\left[A_{\beta}^{+}, \zeta_{b}\right] \mathcal{A}_{b}^{-1} \psi_{b} f
\end{aligned}
$$

where $\left[A_{\beta}^{+}, \zeta_{\nu}\right]=A_{\beta}^{+} \zeta_{\nu}-\zeta_{\nu} A_{\beta}^{+}$for $\nu=a, b$. Since $\partial_{\boldsymbol{n}} \psi_{\nu}=\partial_{\boldsymbol{n}} \zeta_{\nu}=0$ on $\Sigma$, one can prove that $\left[A_{\beta}^{+}, \zeta_{\nu}\right]$ is compact as an operator mapping $\mathscr{V}_{\beta}^{+}(\Omega)$ into $\mathrm{V}_{\beta}^{1}(\Omega)^{*}$. Thus, $\mathrm{R}$ is indeed a right regularizer and coker $A_{\beta}^{+}$is finite-dimensional. 
Step 2: The index of $A_{\beta}^{+}$is equal to zero. By definition, one has $\operatorname{ind}\left(A_{\beta}^{+}\right)=\operatorname{dim}\left(\operatorname{ker} A_{\beta}^{+}\right)-\operatorname{dim}\left(\operatorname{coker} A_{\beta}^{+}\right)$. Define $A_{\beta}: \mathrm{V}_{\beta}^{1}(\Omega) \rightarrow \mathrm{V}_{-\beta}^{1}(\Omega)^{*}$ and $A_{-\beta}: \mathrm{V}_{-\beta}^{1}(\Omega) \rightarrow \mathrm{V}_{\beta}^{1}(\Omega)^{*}$ the two continuous operators such that

$$
\begin{aligned}
\left\langle A_{\beta} u, v\right\rangle_{\Omega} & =(\sigma \nabla u, \nabla v)_{\Omega}, & & \forall(u, v) \in \mathrm{V}_{\beta}^{1}(\Omega) \times \mathrm{V}_{-\beta}^{1}(\Omega) ; \\
\left\langle A_{-\beta} u, v\right\rangle_{\Omega} & =(\sigma \nabla u, \nabla v)_{\Omega}, & & \forall(u, v) \in \mathrm{V}_{-\beta}^{1}(\Omega) \times \mathrm{V}_{\beta}^{1}(\Omega) .
\end{aligned}
$$

Our goal is to prove that $\operatorname{dim}\left(\operatorname{ker} A_{\beta}^{+}\right)=\operatorname{dim}\left(\operatorname{ker} A_{-\beta}\right)$ and $\operatorname{dim}\left(\operatorname{coker} A_{\beta}^{+}\right)=$ $\operatorname{dim}\left(\operatorname{coker} A_{\beta}\right)$. Since $A_{\beta}^{*}=A_{-\beta}$, this will be sufficient to conclude. To begin with, note that $\operatorname{ker} A_{-\beta} \subset \operatorname{ker} A_{\beta}^{+} \subset \operatorname{ker} A_{\beta}$.

i) Consider $u=c s^{+}+u_{-\beta}$, with $c \in \mathbb{C}$ and $u_{-\beta} \in \mathrm{V}_{-\beta}^{1}(\Omega)$, an element of ker $A_{\beta}^{+}$. One has $\operatorname{div}(\sigma \nabla u)=0$ a.e. in $\Omega$. Multiplying by $\bar{u}$, integrating by parts on $\Omega \backslash \overline{\mathrm{B}}(O, \delta)$, taking the limit of the imaginary part when $\delta$ tends to zero (use the ideas of the proof of Proposition 4.1), one finds $c=0$. Consequently, $\operatorname{ker} A_{\beta}^{+}=\operatorname{ker} A_{-\beta}$.

ii) Now, let us prove that $\operatorname{ker} A_{-\beta} \neq \operatorname{ker} A_{\beta}$. Proceed by contradiction assuming temporarily that ker $A_{-\beta}=\operatorname{ker} A_{\beta}$. Introduce $\mathrm{F}$ and $\mathrm{G}$ two finite dimensional vector spaces such that

$$
\begin{aligned}
\mathrm{V}_{\beta}^{1}(\Omega)^{*} & =\operatorname{range} A_{-\beta} \oplus \mathrm{F} \\
\mathrm{F} & =\left(\mathrm{F} \cap \text { range } A_{\beta}\right) \oplus \mathrm{G} .
\end{aligned}
$$

According to Lemma 4.2, proved later, we have $\mathrm{V}_{-\beta}^{1}(\Omega)^{*}=\operatorname{range} A_{\beta}+\mathrm{V}_{\beta}^{1}(\Omega)^{*}$. Consequently, we can write $\mathrm{V}_{-\beta}^{1}(\Omega)^{*}=\operatorname{range} A_{\beta} \oplus \mathrm{G}$. Since $A_{-\beta}$ is the adjoint of $A_{\beta}$, one has $\operatorname{dim}(\mathrm{F})=\operatorname{dim}\left(\operatorname{coker} A_{-\beta}\right)=\operatorname{dim}\left(\operatorname{ker} A_{\beta}\right)$ and $\operatorname{dim}(\mathrm{G})=\operatorname{dim}\left(\operatorname{coker} A_{\beta}\right)=$ $\operatorname{dim}\left(\operatorname{ker} A_{-\beta}\right)$. Thus, our hypothesis leads to $\operatorname{dim}(\mathrm{F})=\operatorname{dim}(\mathrm{G})$ which implies $\mathrm{F} \cap$ range $A_{\beta}=\{0\}$ according to (4.31). Now, recall that $A_{\beta}\left(s^{+}\right) \in \mathrm{V}_{\beta}^{1}(\Omega)^{*}$. According to the decomposition (4.30), there exist $u_{-\beta} \in \mathrm{V}_{-\beta}^{1}(\Omega)$ and $w \in \mathrm{F}$ such that $A_{\beta} s^{+}=A_{-\beta} u_{-\beta}+w$. But clearly $w \in \mathrm{F} \cap \operatorname{range} A_{\beta}$ so $w=0$ and $s^{+}-u_{-\beta} \in \operatorname{ker} A_{\beta}=\operatorname{ker} A_{-\beta}$. It is absurd because $s^{+}-u_{-\beta} \notin \mathrm{V}_{-\beta}^{1}(\Omega)$. Therefore, $\operatorname{ker} A_{-\beta} \not \supset \operatorname{ker} A_{\beta}$.

iii) Consider $u_{\star} \in \operatorname{ker} A_{\beta}$ such that $u_{\star} \notin \operatorname{ker} A_{-\beta}$. Using Theorem 5.2 in annex, one can prove that $u_{\star}$ admits the representation $u_{\star}=c_{\star}^{+} s^{+}+c_{\star}^{-} s^{-}+u_{\star,-\beta}$ with $u_{\star,-\beta} \in \mathrm{V}_{-\beta}^{1}(\Omega)$. Since $u_{\star} \notin \operatorname{ker} A_{-\beta}$, there holds $\left|c_{\star}^{+}\right|+\left|c_{\star}^{-}\right| \neq 0$. On the other hand, proceeding as in $i$ ), one finds that both $c_{\star}^{+}$and $c_{\star}^{-}$are non trivial.

iv) Let us establish that $\operatorname{dim}\left(\operatorname{coker} A_{\beta}^{+}\right)=\operatorname{dim}\left(\operatorname{coker} A_{\beta}\right)$, which will conclude the proof. As in ii), we introduce $\tilde{\mathrm{F}}, \tilde{\mathrm{G}}$ two finite dimensional vector spaces such that

$$
\begin{aligned}
\mathrm{V}_{\beta}^{1}(\Omega)^{*} & =\operatorname{range} A_{\beta}^{+} \oplus \tilde{\mathrm{F}} ; \\
\tilde{\mathrm{F}} & =\left(\tilde{\mathrm{F}} \cap \operatorname{range} A_{\beta}\right) \oplus \tilde{\mathrm{G}} .
\end{aligned}
$$


Again, one has $\mathrm{V}_{-\beta}^{1}(\Omega)^{*}=\operatorname{range} A_{\beta} \oplus \tilde{\mathrm{G}}, \operatorname{dim}(\tilde{\mathrm{F}})=\operatorname{dim}\left(\operatorname{coker} A_{\beta}^{+}\right)$and $\operatorname{dim}(\tilde{\mathrm{G}})=$ $\operatorname{dim}\left(\operatorname{coker} A_{\beta}\right)$. Let us prove that $\tilde{\mathrm{F}}=\tilde{\mathrm{G}}$. According to (4.32), this is equivalent to $\tilde{\mathrm{F}} \cap$ range $A_{\beta}=\{0\}$. Let $f$ be an element of $\tilde{\mathrm{F}} \cap$ range $A_{\beta}$. There exists $u \in \mathrm{V}_{\beta}^{1}(\Omega)$ such that $f=A_{\beta} u$. Using Theorem 5.2 in annex, one finds that $u$ admits the representation $u=c^{+} s^{+}+c^{-} s^{-}+u_{-\beta}$ with $u_{-\beta} \in \mathrm{V}_{-\beta}^{1}(\Omega)$. Now, observing that $u-c^{-} u_{\star} / c_{\star}^{-}$is an element of $\mathscr{V}_{\beta}^{+}(\Omega)$ such that $A_{\beta}^{+}\left(u-c^{-} u_{\star} / c_{\star}^{-}\right)=f$, one deduces that $f \in \tilde{\mathrm{F}} \cap$ range $A_{\beta}^{+}$and, since $\tilde{\mathrm{F}} \cap$ range $A_{\beta}^{+}=\{0\}$, we have $f=0$.

Lemma 4.2. $\mathrm{V}_{-\beta}^{1}(\Omega)^{*}=\operatorname{range} A_{\beta}+\mathrm{V}_{\beta}^{1}(\Omega)^{*}$.

Proof. Consider $f \in \mathrm{V}_{-\beta}^{1}(\Omega)^{*}$, and take $\psi_{a}$ and $\omega_{a}$ like in the proof of Proposition 4.4 above. The function $\psi_{a} f$ belongs to $\mathrm{V}_{-\beta}^{1}\left(\omega_{a}\right)^{*}$. Since $\mathcal{A}_{a \beta}$, the unique linear continuous operator from $\mathrm{V}_{\beta}^{1}\left(\omega_{a}\right)$ to $\mathrm{V}_{-\beta}^{1}\left(\omega_{a}\right)^{*}$ satisfying $\left\langle\mathcal{A}_{a \beta} u, v\right\rangle_{\omega_{a}}=(\sigma \nabla u, \nabla v)_{\omega_{a}}$, $\forall u \in \mathrm{V}_{\beta}^{1}\left(\omega_{a}\right), \forall v \in \mathrm{V}_{-\beta}^{1}\left(\omega_{a}\right)$, is onto (see the discussion after Lemma 4.1), we know there exists $u \in \mathrm{V}_{\beta}^{1}\left(\omega_{a}\right)$ such that $\mathcal{A}_{a \beta} u=\psi_{a} f$. The function $A_{\beta}\left(\zeta_{a} u\right) \in \operatorname{range} A_{\beta}$ is such that $f-A_{\beta}\left(\zeta_{a} u\right) \in \mathrm{V}_{\beta}^{1}(\Omega)^{*}$.

Remark 4.6. Up to now, we have considered a static problem, but, as mentioned in section 2, similar results can be obtained for the time harmonic problem, with a pulsation $\omega_{0}>0$. Following ${ }^{21}$ and going back to the time-domain, we observe that our results lead to consider solutions which behave like

$$
e^{i\left(\eta_{\sigma} \ln r-\omega_{0} t\right)} \varphi_{p}(\theta)
$$

near the origin. Two surprising properties can be noticed.

1) This outgoing wave seems to come from the origin, that is from the infinity in the strip. This is due to the presence of the metamaterial, in which phase and group velocities are known to be of opposite signs ${ }^{30}$, and is strongly related to the choice of dissipative model used in $\S 4.4$ (see Remark 4.5).

2) The velocity of the wave $\omega_{0} r / \eta_{\sigma}$ tends to 0 when it approaches the origin. Furthermore, when $\kappa_{\sigma} \rightarrow-1$, we have $\eta_{\sigma} \rightarrow+\infty$. Consequently, the closer $\kappa_{\sigma}$ is to -1 , the slower the wave propagates.

\section{Analysis in weighted Sobolev spaces}

The aim of this section is to prove the technical results that were required in the previous section. In particular, we are going to give a detailed proof for Theorem 4.1 and Lemma 4.1. The analysis that we present here is widely inspired by Chapter 6 of ${ }^{12}$. However, we wish to give a detailed presentation of the technical results of this section for two reasons. First, we do not expect our reader to be familiar with this theory, so it seems more appropriate to provide, within this article, all necessary material for a full comprehension. The second reason is that, in the present situation, we need to tackle a problem that is not elliptic, whereas ellipticity is assumed in 
12. So, it is important to check that the results in ${ }^{12}$ can be adapted to treat our particular case. In the sequel, for the sake of conciseness, we set

$$
I \stackrel{\text { def }}{=}(0, \pi) \text {. }
$$

We will denote $(\cdot, \cdot)$ the product of $\mathrm{L}^{2}(I)$ and $(\cdot, \cdot)_{1}$ (resp. $\left.(\cdot, \cdot)_{2}\right)$ the one of $\mathrm{L}^{2}(\pi / 4, \pi)$ (resp. $\left.\mathrm{L}^{2}(0, \pi / 4)\right)$. For a general measurable function $v$ on $(0, \pi)$, we define the restrictions $v_{1}:=\left.v\right|_{(\pi / 4, \pi)}$ and $v_{2}:=\left.v\right|_{(0, \pi / 4)}$.

\subsection{Parameter dependent norm}

In the next paragraph, we will have to study one dimensional problems involving functions in $\mathrm{H}_{0}^{1}(I)$. However, these problems will depend on a complex valued parameter $\lambda$. Since we need to state estimates in which behaviour for $|\lambda|$ large is a key consideration, we shall not consider the standard norm over $\mathrm{H}_{0}^{1}(I)$ but a parameter-dependent norm defined by

$$
\|v\|_{\mathrm{H}^{1}(I,|\lambda|)} \stackrel{\text { def }}{=}\left(\|v\|_{\mathrm{H}^{1}(I)}^{2}+|\lambda|^{2}\|v\|_{\mathrm{L}^{2}(I)}^{2}\right)^{1 / 2}, \quad \forall v \in \mathrm{H}^{1}(I) .
$$

Admittedly, for any fixed $\lambda \in \mathbb{C}$, the norms \|\|$_{\mathrm{H}^{1}(I,|\lambda|)}$ and \|\|$_{\mathrm{H}^{1}(I)}$ are equivalent. But the difference between these two norms comes into play in estimates involving large values of $|\lambda|$. We also consider the dual norm

$$
\|f\|_{\mathrm{H}^{-1}(I,|\lambda|)} \stackrel{\text { def }}{=} \sup _{v \in \mathrm{H}_{0}^{1}(I)} \frac{\left|\langle f, v\rangle_{I}\right|}{\|v\|_{\mathrm{H}^{1}(I,|\lambda|)}}, \quad \forall f \in \mathrm{H}^{-1}(I) .
$$

\subsection{Parameter dependent 1-D problem}

As a preliminary step, we need to study the properties of the operator pencil $\mathscr{L}(\lambda)$ : $\mathrm{H}_{0}^{1}(I) \rightarrow \mathrm{H}^{-1}(I)$, and to state estimates for it in terms of the norms (5.1) and (5.2). Recall that this pencil is defined by

$$
\langle\mathscr{L}(\lambda) u, v\rangle_{I}=\int_{0}^{\pi} \sigma \partial_{\theta} u \partial_{\theta} v d \theta-\lambda^{2} \int_{0}^{\pi} \sigma u v d \theta, \quad \forall u, v \in \mathrm{H}_{0}^{1}(I) .
$$

Clearly $\mathscr{L}(\lambda)$ has analytic dependency with respect to $\lambda$ in the norm of continuous operators mapping $\mathrm{H}_{0}^{1}(I)$ into $\mathrm{H}^{-1}(I)$. Injectivity of $\mathscr{L}(\lambda)$ has already been investigated in sub-section 4.1 by means of direct calculus. We would like to derive estimates for the behaviour of $\|\mathscr{L}(\lambda) u\|_{\mathrm{H}^{-1}(I)}$ for $|\lambda|$ large.

Lemma 5.1. Assume that $\kappa_{\sigma}=\sigma_{2} / \sigma_{1} \neq-1$. Then, $\mathscr{L}(\lambda)$ is an isomorphism from $\mathrm{H}_{0}^{1}(I)$ to $\mathrm{H}^{-1}(I)$ if and only if there holds $\lambda \notin \Lambda\left(\kappa_{\sigma}\right)$, where $\Lambda\left(\kappa_{\sigma}\right)$ is the set of eigenvalues of $\mathscr{L}$.

Proof. As in the proof of Lemma 3.1, we use the technique of $T$-coercivity. To obtain this result, we will first prove the

Lemma 5.2. Assume that $\kappa_{\sigma} \neq-1$. Then, there exists $\tau_{0} \in \mathbb{R}$ such that $\mathscr{L}\left(i \tau_{0}\right)$ is an isomorphism from $\mathrm{H}_{0}^{1}(I)$ to $\mathrm{H}^{-1}(I)$. 
Proof. As in the proof of Lemma 3.1, we use the technique of $T$-coercivity. Consider a $\mathscr{C}^{\infty}$ cut-off function $\psi: \mathbb{R} \rightarrow[0,1]$ such that $\psi(z)=1$ for $|z-\pi / 4|<\pi / 16$ and $\psi(z)=0$ for $|z-\pi / 4|>\pi / 8$. Introduce the isomorphisms $T_{1}, T_{2}: \mathrm{H}_{0}^{1}(I) \rightarrow \mathrm{H}_{0}^{1}(I)$ defined by

$$
\begin{aligned}
& \left(T_{1} v\right)(\theta)=\left\{\begin{array}{ll}
v_{1}(\theta) & \text { for } \theta \in(\pi / 4, \pi) \\
-v_{2}(\theta)+2 \psi(\theta) v_{1}(\pi / 2-\theta) & \text { for } \theta \in(0, \pi / 4)
\end{array},\right. \\
& \left(T_{2} v\right)(\theta)=\left\{\begin{array}{ll}
v_{1}(\theta)-2 \psi(\theta) v_{2}(\pi / 2-\theta) & \text { for } \theta \in(\pi / 4, \pi) \\
-v_{2}(\theta) & \text { for } \theta \in(0, \pi / 4)
\end{array} .\right.
\end{aligned}
$$

for all $v \in \mathrm{H}_{0}^{1}(I)$. Denote $s(\theta)=\pi / 2-\theta$. For all $\lambda=i \tau$ with $\tau \in \mathbb{R}$, one has, for all $\alpha, \delta>0$ and $v \in \mathrm{H}_{0}^{1}(I)$,

$$
\begin{aligned}
\left|\left\langle\mathscr{L}(i \tau) v, T_{1} \bar{v}\right\rangle_{I}\right| & \\
\geq & \sigma_{1}\left(v_{1}^{\prime},{\overline{v_{1}}}^{\prime}\right)_{1}+\tau^{2} \sigma_{1}\left(v_{1}, \overline{v_{1}}\right)_{1}+\left|\sigma_{2}\right|\left(v_{2}^{\prime},{\overline{v_{2}}}^{\prime}\right)_{2}+\tau^{2}\left|\sigma_{2}\right|\left(v_{2}, \overline{v_{2}}\right)_{2} \\
& -2\left|\sigma_{2}\left(v_{2}^{\prime},\left(\psi \overline{v_{1}} \circ s\right)^{\prime}\right)_{2}\right|-2\left|\tau^{2} \sigma_{2}\left(v_{2},\left(\psi \overline{v_{1}} \circ s\right)\right)_{2}\right| \\
\geq & \left(\sigma_{1}-\alpha^{-1}\left|\sigma_{2}\right|\right)\left(v_{1}^{\prime},{\overline{v_{1}}}^{\prime}\right)_{1}+(1-(\alpha+\delta))\left|\sigma_{2}\right|\left(v_{2}^{\prime},{\overline{v_{2}}}^{\prime}\right)_{2} \\
\quad & +\left(\tau^{2}\left(\sigma_{1}-\alpha^{-1}\left|\sigma_{2}\right|\right)-\delta^{-1} C\left|\sigma_{2}\right|\right)\left(v_{1}, \overline{v_{1}}\right)_{1}+\left(\tau^{2}\left|\sigma_{2}\right|(1-\alpha)\right)\left(v_{2}, \overline{v_{2}}\right)_{2},
\end{aligned}
$$

with $C=\sup _{I}\left|\psi^{\prime}\right|$. Suppose that $\sigma_{1}>\left|\sigma_{2}\right|$. One can find $\alpha>0$ such that $\sigma_{1}-$ $\alpha^{-1}\left|\sigma_{2}\right|>0$ and $1-\alpha>0$. Next, choose $\delta>0$ such that $\delta<1-\alpha$. From (5.4), one deduces that there exist two constants $C_{1}, C_{2}>0$, independent of $\tau$, such that $\left|\left\langle\mathscr{L}(i \tau) v, T_{1} \bar{v}\right\rangle_{I}\right| \geq C_{1}\left(\left(v^{\prime}, \bar{v}^{\prime}\right)+\tau^{2}(v, \bar{v})\right)-C_{2}(v, \bar{v})$ for all $v \in \mathrm{H}_{0}^{1}(I)$. This proves that for $\tau^{2}$ large enough, $(v, w) \mapsto\left\langle\mathscr{L}(i \tau) v, T_{1} \bar{w}\right\rangle_{I}$ is coercive. One concludes as in the proof of Lemma 3.1 that $\mathscr{L}(i \tau)$ is an isomorphism from $\mathrm{H}_{0}^{1}(I)$ to $\mathrm{H}^{-1}(I)$ for $\tau^{2}$ sufficiently large. When $\left|\sigma_{2}\right|>\sigma_{1}$, one proceeds in the same way working with $T_{2} \cdot$

Coming back to the proof of Lemma 5.1 , let $\tau_{0} \in \mathbb{R}$ be given by Lemma 5.2 such that $\mathscr{L}\left(i \tau_{0}\right)$ is an isomorphism. For all $\lambda \in \mathbb{C}, \mathscr{L}(\lambda)-\mathscr{L}\left(i \tau_{0}\right)$ is a compact operator from $\mathrm{H}_{0}^{1}(I)$ to $\mathrm{H}^{-1}(I)$. Fredholm alternative then ensures that $\mathscr{L}(\lambda)$ is an isomorphism if, and only if, $\lambda$ is not an eigenvalue of $\mathscr{L}$.

According to Theorem 1.1.1 in ${ }^{13}$, the preceding result shows that, for $\kappa_{\sigma} \neq-1$, the set $\Lambda\left(\kappa_{\sigma}\right)$ of eigenvalues of $\mathscr{L}$ is discrete and $\mathscr{L}(\lambda)^{-1}$ is well-defined and analytic in $\mathbb{C} \backslash \Lambda\left(\kappa_{\sigma}\right)$.

Proposition 5.1. Assume that $\kappa_{\sigma}=\sigma_{2} / \sigma_{1} \neq-1$. Let $\beta \in \mathbb{R}$ be such that no eigenvalue $\lambda$ of $\mathscr{L}(\lambda)$ satisfies $\Re e\{\lambda\}=\beta$. Then, there exists a constant $C_{\beta}>0$ independent of $\lambda$ such that

$$
\|u\|_{\mathrm{H}^{1}(I,|\lambda|)} \leq C_{\beta}\|\mathscr{L}(\lambda) u\|_{\mathrm{H}^{-1}(I,|\lambda|)}, \quad \forall u \in \mathrm{H}_{0}^{1}(I),
$$

for all $\lambda=\beta+i \mu, \mu \in \mathbb{R}$. 
Proof. We will prove this proposition for $\sigma_{1}>\left|\sigma_{2}\right|$. The case $\left|\sigma_{2}\right|>\sigma_{1}$ can be studied in the same manner working with $T_{2}$ instead of $T_{1}$. Consider first $\lambda=i \tau \in$ $i \mathbb{R}$. The estimate (5.4) proves that there exists $r_{0}>0$ such that if $|\tau|>r_{0}$, then

$$
\left\langle\mathscr{L}(i \tau) v, T_{1} \bar{v}\right\rangle_{I} \geq C_{1}\left(\left(v^{\prime}, \bar{v}^{\prime}\right)+\tau^{2}(v, \bar{v})\right),
$$

for all $v \in \mathrm{H}_{0}^{1}(I)$, where $C_{1}$ is a constant independent of $\tau$. Since the operator $T_{1}$ defined in (5.3) is also continuous from $\mathrm{L}^{2}(I)$ into $\mathrm{L}^{2}(I)$, one obtains (5.5) for $\lambda=i \tau \in i \mathbb{R}$ with $|\tau|>r_{0}$.

Consider now the case $\lambda=i \tau e^{i \phi}$ with $\phi \in[-\pi / 2 ; \pi / 2]$. Here, $\tau \in \mathbb{R}$ is such that $|\tau|>r_{0}$ with $r_{0}$ defined above. One has, for all $v \in \mathrm{H}_{0}^{1}(I)$,

$$
\left|\left\langle\mathscr{L}(\lambda) v, T_{1} \bar{v}\right\rangle_{I}-\left\langle\mathscr{L}(i \tau) v, T_{1} \bar{v}\right\rangle_{I}\right| \leq C_{2} \tau^{2}\left|1-e^{2 i \phi}\right|(v, \bar{v}),
$$

where $C_{2}$ is a constant independent of $\tau$. Combining (5.6) and (5.7), one finds

$$
\begin{aligned}
\left|\left\langle\mathscr{L}(\lambda) v, T_{1} \bar{v}\right\rangle_{I}\right| & \geq\left|\left\langle\mathscr{L}(i \tau) v, T_{1} \bar{v}\right\rangle_{I}\right|-C_{2} \tau^{2}\left|1-e^{2 i \phi}\right|(v, \bar{v}) \\
& \geq C_{1}\left(v^{\prime}, \bar{v}^{\prime}\right)+\tau^{2}\left(C_{1}-C_{2}\left|1-e^{2 i \phi}\right|\right)(v, \bar{v}) .
\end{aligned}
$$

Taking $\phi$ small enough to have, for example, $C_{2}\left|1-e^{2 i \phi}\right| \leq C_{1} / 2$, one deduces there exist two real constants $r_{0}$ and $\delta_{0}$ such that (5.5) holds true for all $\lambda \in \mathbb{C}$ satisfying $|\lambda|>r_{0}$ and $|\Re e\{\lambda\}|<\delta_{0}|\Im m\{\lambda\}|$.

Finally, since $\mathscr{L}(\lambda)^{-1}$ is well-defined and analytic in a neighbourhood of the line $\Re e\{\lambda\}=\beta$, one can state the result of the proposition.

\subsection{Weighted spaces and Fourier transform in the full strip}

In this paragraph, using Fourier-Laplace transform, we will study the relationship between the space $\mathrm{H}_{0}^{1}(I)$ and $\mathrm{H}^{-1}(I)$, and weighted Sobolev spaces in a "full strip". Define

$$
\mathcal{S} \stackrel{\text { def }}{=} \mathbb{R} \times(0, \pi) .
$$

Let us introduce a family of weighted spaces over this set. For any $\beta \in \mathbb{R}$ and $k \in \mathbb{N}$, the space $\mathrm{W}_{\beta}^{k}(\mathcal{S})$ will refer to the completion of $\mathscr{C}_{0}^{\infty}(\mathcal{S})$ with respect to the weighted norm defined by

$$
\|v\|_{\mathrm{W}_{\beta}^{k}(\mathcal{S})} \stackrel{\text { def }}{=}\left(\sum_{\alpha+\gamma \leq k}\left\|e^{\beta z} \partial_{z}^{\alpha} \partial_{\theta}^{\gamma} v\right\|_{\mathrm{L}^{2}(\mathcal{S})}^{2}\right)^{1 / 2} .
$$

We shall denote $\mathrm{W}_{\beta}^{1}(\mathcal{S})^{*}$ the topological dual to the space $\mathrm{W}_{\beta}^{1}(\mathcal{S})$. The norm over $\mathrm{W}_{\beta}^{1}(\mathcal{S})^{*}$ will be defined by

$$
\|f\|_{\mathrm{W}_{\beta}^{1}(\mathcal{S})^{*}} \stackrel{\text { def }}{=} \sup _{v \in \mathrm{W}_{\beta}^{1}(\mathcal{S})} \frac{\left|\langle f, v\rangle_{\mathcal{S}}\right|}{\|v\|_{\mathrm{W}_{\beta}^{1}(\mathcal{S})}}, \quad \forall f \in \mathrm{W}_{\beta}^{1}(\mathcal{S})^{*} .
$$


In order to study the relationship between these weighted spaces and the spaces that we introduced in the preceding paragraph, we make intensive use of the FourierLaplace transform. This transform is defined by a density process from the following identity

$$
\widehat{v}(\lambda, \theta) \stackrel{\text { def }}{=} \int_{\mathbb{R}} v(z, \theta) e^{-\lambda z} d z, \quad \forall v \in \mathscr{C}_{0}^{\infty}(\mathcal{S}), \quad \forall \lambda \in \mathbb{C} .
$$

It is also possible to define the Fourier-Laplace transform of elements of $\mathrm{H}^{-1}(\mathcal{S})$, the dual space to $\mathrm{H}_{0}^{1}(\mathcal{S})$. For the sake of conciseness, we restrict our definition to compactly supported elements (although this definition can be extended). Assume that $f \in \mathrm{H}^{-1}(\mathcal{S})$ has a compact support. Then its partial Fourier transform with respect to the variable $z$ will refer to the function $\lambda \mapsto \widehat{f}(\lambda, \cdot)$, valued in $\mathrm{H}^{-1}(I)$, defined by

$$
\langle\widehat{f}(\lambda), v\rangle_{I}=\left\langle f, \widetilde{v}_{\lambda}\right\rangle_{\mathcal{S}} \quad \text { with } \quad \widetilde{v}_{\lambda}(z, \theta)=e^{-\lambda z} v(\theta), \quad \forall v \in \mathrm{H}_{0}^{1}(I) .
$$

Although $\widetilde{v}_{\lambda} \notin \mathrm{H}_{0}^{1}(\mathcal{S})$, the identity above makes sense because $f$ has compact support. As a function valued in $\mathrm{H}^{-1}(I)$, the function $\widehat{f}$ admits analytical dependency with respect to $\lambda$.

Note that, for $v \in \mathrm{W}_{\beta}^{1}(\mathcal{S}), \beta \in \mathbb{R},(\xi, \theta) \mapsto \widehat{v}(-\beta+i \xi, \theta)$ is actually the (partial) Fourier transform with respect to $z$ of $(z, \theta) \mapsto e^{\beta z} u(z, \theta)$. Using this observation, as well as the classical characterization of Sobolev spaces by means of Fourier transform, it is straightforward to prove the following Parseval identity

$$
\|v\|_{\mathrm{W}_{\beta}^{1}(\mathcal{S})}^{2}=\frac{1}{2 i \pi} \int_{-\beta-i \infty}^{-\beta+i \infty}\|\widehat{v}(\lambda, \cdot)\|_{\mathrm{H}^{1}(I,|\lambda|)}^{2} d \lambda, \quad \forall v \in \mathscr{C}_{0}^{\infty}(\mathcal{S}), \quad \forall \beta \in \mathbb{R} .
$$

A similar relationship holds between $\mathrm{W}_{\beta}^{1}(\mathcal{S})^{*}$ and $\mathrm{H}^{1}(I)$. This is the precise reason why we introduced the parameter dependent norms \|\|$_{\mathrm{H}^{1}(I,|\lambda|)}$ and \|\|$_{\mathrm{H}^{-1}(I,|\lambda|)}$ (such a Parseval identity would not hold with the standard norm \|\|$_{\mathrm{H}^{-1}(I)}$ ).

Lemma 5.3. Let $f \in \mathrm{H}^{-1}(\mathcal{S})$ have compact support. Then we have

$$
\|f\|_{\mathrm{W}_{\beta}^{1}(\mathcal{S})^{*}}^{2}=\frac{1}{2 i \pi} \int_{\beta-i \infty}^{\beta+i \infty}\|\widehat{f}(\lambda, \cdot)\|_{\mathrm{H}^{-1}(I,|\lambda|)}^{2} d \lambda, \quad \forall \beta \in \mathbb{R} .
$$

Proof. Takes $\beta \in \mathbb{R}$. First of all, note that $(u, v) \mapsto\left(e^{2 \beta z} \nabla u, \nabla \bar{v}\right)_{\mathcal{S}}+\left(e^{2 \beta z} u, \bar{v}\right)_{\mathcal{S}}$ is a scalar product for $\mathrm{W}_{\beta}^{1}(\mathcal{S})$. Consider a functional $f \in \mathrm{H}^{-1}(\mathcal{S})$ such that, for some $u \in \mathscr{C}_{0}^{\infty}(\mathcal{S})$, we have

$$
\langle f, w\rangle_{I}=\left(e^{2 \beta z} \nabla u, \nabla w\right)_{\mathcal{S}}+\left(e^{2 \beta z} u, w\right)_{\mathcal{S}} \quad \forall w \in \mathrm{W}_{\beta}^{1}(\mathcal{S}) .
$$

Since $\mathscr{C}_{0}^{\infty}(\mathcal{S})$ is dense in $\mathrm{W}_{\beta}^{1}(\mathcal{S})$, according to Riesz Theorem, functionals of the form $(5.10)$ with $u \in \mathscr{C}_{0}^{\infty}(\mathcal{S})$ are dense in $\mathrm{W}_{\beta}^{1}(\mathcal{S})^{*}$ for \|\|$_{\mathrm{W}_{\beta}^{1}(\mathcal{S})^{*}}$. Hence it is sufficient to prove (5.9) only for such functionals. 
Observe that (5.10) still makes sense whenever $w(z, \theta)=e^{-\lambda z} v(\theta)$ for any $\lambda=$ $\beta+i \eta \in \mathbb{C}$, since $u$ is compactly supported. Taking such a $w(z, \theta)$, we obtain

$$
\langle\widehat{f}(\beta+i \eta), v\rangle_{I}=\left(\partial_{\theta} \widehat{u}(-\beta+i \eta), \partial_{\theta} v\right)_{I}+\left(1+|\beta+i \eta|^{2}\right)(\widehat{u}(-\beta+i \eta), v)_{I}
$$

for all $\eta \in \mathbb{R}$ and all $v \in \mathrm{H}_{0}^{1}(I)$. For a fixed $\eta \in \mathbb{R}$, taking $v(\theta)=\bar{u}(-\beta+i \eta, \theta)$, we obtain the identity

$$
\|\widehat{f}(\beta+i \eta)\|_{\mathrm{H}^{-1}(I,|\beta+i \eta|)}^{2}=\|\widehat{u}(-\beta+i \eta)\|_{\mathrm{H}^{-1}(I,|-\beta+i \eta|)}^{2} \quad \forall \eta \in \mathbb{R} .
$$

Now we come back to $(5.10)$, take $w(z, \theta)=\bar{u}(z, \theta)$, and apply Parseval identity (5.8), which yields

$$
\langle f, \bar{u}\rangle_{\mathcal{S}}=\frac{1}{2 i \pi} \int_{-\beta-i \infty}^{-\beta+i \infty}\|\widehat{u}(\lambda, \cdot)\|_{\mathrm{H}^{1}(I,|\lambda|)}^{2} d \lambda=\frac{1}{2 i \pi} \int_{\beta-i \infty}^{\beta+i \infty}\|\widehat{f}(\lambda, \cdot)\|_{\mathrm{H}^{1}(I,|\lambda|)}^{2} d \lambda
$$

As is readily checked, we have $\langle f, \bar{u}\rangle_{\mathcal{S}}=\|f\|_{\mathrm{W}^{1}(\mathcal{S})^{*}}^{2}$. Plugging this in the previous equation concludes the proof.

\subsection{Problem in the full strip}

In the present section we study the invertibility of some operator $G_{\beta}$ defined in the full strip. For any $\beta \in \mathbb{R}$, define $G_{\beta}$ as the unique continuous linear operator mapping $\mathrm{W}_{\beta}^{1}(\mathcal{S})$ into $\mathrm{W}_{-\beta}^{1}(\mathcal{S})^{*}$ such that

$$
\left\langle G_{\beta} u, v\right\rangle_{\mathcal{S}}=(\sigma \nabla u, \nabla v)_{\mathcal{S}}, \quad \forall u \in \mathrm{W}_{\beta}^{1}(\mathcal{S}), \quad \forall v \in \mathrm{W}_{-\beta}^{1}(\mathcal{S}) .
$$

Theorem 5.1. Assume that $\kappa_{\sigma}=\sigma_{2} / \sigma_{1} \neq-1$. For $\beta \in \mathbb{R}$, the operator $G_{\beta}: \mathrm{W}_{\beta}^{1}(\mathcal{S}) \rightarrow \mathrm{W}_{-\beta}^{1}(\mathcal{S})^{*}$ is an isomorphism if no eigenvalue $\lambda$ of the pencil $\mathscr{L}(\lambda)$ satisfies $\Re e\{\lambda\}=\beta$.

Proof. As a first step we show that, for any $\beta \in \mathbb{R}$ such that no eigenvalue $\lambda$ of $\mathscr{L}(\lambda)$ satisfies $\Re e\{\lambda\}=\beta$, there exists a constant $C_{\beta}>0$ such that

$$
\|u\|_{\mathrm{W}_{\beta}^{1}(\mathcal{S})} \leq C_{\beta}\left\|G_{\beta} u\right\|_{\mathrm{W}_{-\beta}^{1}(\mathcal{S})^{*}}, \quad \forall u \in \mathrm{W}_{\beta}^{1}(\mathcal{S}) .
$$

Since $\mathscr{C}_{0}^{\infty}(\mathcal{S})$ is dense in $\mathrm{W}_{\beta}^{1}(\mathcal{S})$, it suffices to show that such an inequality holds for any $u$ chosen in $\mathscr{C}_{0}^{\infty}(\mathcal{S})$. Take any $u \in \mathscr{C}_{0}^{\infty}(\mathcal{S})$, and observe that

$$
\widehat{G_{\beta} u}(\lambda, \theta)=\mathscr{L}(\lambda) \widehat{u}(\lambda, \theta), \quad \forall \lambda \in \mathbb{C} .
$$

Inequality (5.11) is then a direct consequence of Proposition 5.1 and Lemma 5.3. Inequality (5.11) proves that $G_{\beta}$ is a one-to-one operator with closed range. Moreover, we have $G_{\beta}^{*}=G_{-\beta}$, and no eigenvalue of $\mathscr{L}(\lambda)$ satisfies $\Re e\{\lambda\}=-\beta$ since none of them satisfies $\Re e\{\lambda\}=\beta$, see Formula (4.8). As a consequence $G_{\beta}^{*}$ is also one-to-one, as (5.11) still holds with $\beta$ replaced by $-\beta$, and since range $G_{\beta}$ is closed, this implies that $G_{\beta}$ is onto, see Theorem 2.13 in ${ }^{17}$ for example. 


\subsection{Problem in the half strip}

Now, we come to study problems set on the half strip $\mathcal{B}=(-\infty, 0) \times(0, \pi)$, which was the geometry we had to consider in Section 4. Recall that we have defined the continuous operators $L_{\beta}: \mathrm{W}_{\beta}^{1}(\mathcal{B}) \rightarrow \mathrm{W}_{-\beta}^{1}(\mathcal{B})^{*}$ such that

$$
\left\langle L_{\beta} u, v\right\rangle_{\mathcal{B}}=(\sigma \nabla u, \nabla v)_{\mathcal{B}}, \quad \forall u \in \mathrm{W}_{\beta}^{1}(\mathcal{B}), \quad \forall v \in \mathrm{W}_{-\beta}^{1}(\mathcal{B}) .
$$

Theorem 4.1. Assume that $\kappa_{\sigma}=\sigma_{2} / \sigma_{1} \neq-1$. The operator $L_{\beta}: \mathrm{W}_{\beta}^{1}(\mathcal{B}) \rightarrow$ $\mathrm{W}_{-\beta}^{1}(\mathcal{B})^{*}$ is of Fredholm type if and only if no eigenvalue of $\mathscr{L}(\lambda)$ belongs

$$
\ell_{\beta} \stackrel{\text { def }}{=}\{\lambda \in \mathbb{C} \mid \Re e\{\lambda\}=\beta\} .
$$

Proof. Take any $\beta \in \mathbb{R}$ and assume first that no eigenvalue of $\mathscr{L}(\lambda)$ satisfies $\Re e\{\lambda\}=\beta$. Set $Q=(-2,0) \times(0, \pi)$. Consider a $\mathscr{C}^{\infty}$ cut-off function $\zeta: \mathbb{R} \rightarrow[0,1]$ such that $\zeta(z)=0$ for $z \geq-1$ and $\zeta(z)=1$ for $z \leq-2$, and set $\chi(z)=1-\zeta(z)$. According to Theorem 3.3 and estimate $(7)$ in ${ }^{1}$, there exists a constant $C_{1}>0$ such that

$$
\|v\|_{\mathrm{H}^{1}(Q)} \leq C_{1}\left(\|\operatorname{div}(\sigma \nabla v)\|_{\mathrm{H}^{-1}(Q)}+\|v\|_{\mathrm{L}^{2}(Q)}\right), \quad \forall v \in \mathrm{H}_{0}^{1}(Q) .
$$

Note that $\operatorname{div}(\sigma \nabla(\chi u))=\chi \operatorname{div}(\sigma \nabla u)+2 \operatorname{div}(u \sigma \nabla \chi)-u \sigma \partial_{z}^{2} \chi$ belongs to $\mathrm{H}^{-1}(Q)$ for all $u \in \mathrm{W}_{\beta}^{1}(\mathcal{B})$. Hence, applying Inequality (5.12) to $\chi u$ yields the existence of a constant $C_{2}>0$ such that

$$
\|\chi u\|_{\mathrm{H}^{1}(Q)} \leq C_{2}\left(\left\|L_{\beta} u\right\|_{\mathrm{W}_{-\beta}^{1}(\mathcal{B})^{*}}+\|u\|_{\mathrm{L}^{2}(Q)}\right), \quad \forall u \in \mathrm{W}_{\beta}^{1}(\mathcal{B}) .
$$

Extend $\zeta u$ by 0 in $\mathcal{S} \backslash \bar{B}$ so that $\zeta u$ may be considered as an element of $\mathrm{W}_{\beta}^{1}(\mathcal{S})$. Hence, we can apply Theorem 5.1 to it: there exists a constant $C_{3}$ such that

$$
\|\zeta u\|_{\mathrm{W}_{\beta}^{1}(\mathcal{S})} \leq C_{3}\left\|G_{\beta}(\zeta u)\right\|_{\mathrm{W}_{-\beta}^{1}(\mathcal{S})^{*}}, \quad \forall u \in \mathrm{W}_{\beta}^{1}(\mathcal{B}) .
$$

Similarly, $\zeta L_{\beta}(u)$ may be considered as an element of $\mathrm{W}_{-\beta}^{1}(\mathcal{S})^{*}$. Since $G_{\beta}(\zeta u)=$ $\zeta L_{\beta}(u)+2 \operatorname{div}(u \sigma \nabla \zeta)-u \sigma \partial_{z}^{2} \zeta$, we obtain, for all $u \in \mathrm{W}_{\beta}^{1}(\mathcal{B})$,

$$
\|\zeta u\|_{\mathrm{W}_{\beta}^{1}(\mathcal{B})}=\|\zeta u\|_{\mathrm{W}_{\beta}^{1}(\mathcal{S})} \leq C_{3}^{\prime}\left(\left\|L_{\beta} u\right\|_{\mathrm{W}_{-\beta}^{1}(\mathcal{B})^{*}}+\|u\|_{\mathrm{L}^{2}(Q)}\right) .
$$

Since $\zeta+\chi=1$, gathering inequalities (5.13) and (5.14), we deduce that there exists a constant $C_{4}>0$ such that

$$
\|u\|_{\mathrm{W}_{\beta}^{1}(\mathcal{B})} \leq C_{4}\left(\left\|L_{\beta} u\right\|_{\mathrm{W}_{-\beta}^{1}(\mathcal{B})^{*}}+\|u\|_{\mathrm{L}^{2}(Q)}\right), \quad \forall u \in \mathrm{W}_{\beta}^{1}(\mathcal{B}) .
$$

The restriction $\left.u \mapsto u\right|_{Q}$ from $\mathrm{W}_{\beta}^{1}(\mathcal{B})$ to $\mathrm{L}^{2}(Q)$ is compact. Consequently, Lemma 6.1 applied to inequality (5.15) shows that range $L_{\beta}$ is closed and $\operatorname{ker} L_{\beta}$ has finite dimension.

Moreover, observe that no eigenvalue of $\mathscr{L}(\lambda)$ satisfies $\Re e\{\lambda\}=\beta$ if and only if no eigenvalue of $\mathscr{L}(\lambda)$ satisfies $\Re e\{\lambda\}=-\beta$. What precedes also holds with $\beta$ replaced by $-\beta$. In particular, since $L_{\beta}^{*}=L_{-\beta}$ we have $\operatorname{dim}\left(\operatorname{coker} L_{\beta}\right)=$ $\operatorname{dim}\left(\operatorname{ker} L_{-\beta}\right)<+\infty$. This finally shows that $L_{\beta}$ is of Fredholm type, and concludes the first part of the proof. 
Now, assume that there exists one eigenvalue $\lambda_{0} \in \mathbb{C}$ of $\mathscr{L}$ such that $\Re e\left\{\lambda_{0}\right\}=\beta$. Take the cut-off function $\chi(z)$ as defined earlier in the proof, and set $u_{n}(z, \theta)=$ $\chi(z / n) \exp \left(\lambda_{0} z\right) \varphi_{\mathrm{p}}(\theta)$ where $\varphi_{p}(\theta)$, defined in (4.9), is an eigenvector of $\mathscr{L}\left(\lambda_{0}\right)$. Taking $n \rightarrow+\infty$ shows that (5.14) cannot hold when applied to the sequence $u_{n}$. Since $\operatorname{dim}\left(\operatorname{ker} L_{\beta}\right)<+\infty$ (observe indeed that $\operatorname{ker} L_{\beta} \subset \operatorname{ker} L_{\gamma}$ for all $\gamma>\beta$ ), this will prove that range $L_{\beta}$ cannot be closed. Otherwise, we could establish estimate (5.14) by classical arguments: one can for instance adapt the proof of lemma 5.1 in chapter 2 of ${ }^{15}$, noticing that $L_{\beta} u=0$ and $u=0$ in $Q$ implies $u=0$ (indeed $G_{\beta^{\prime}} \tilde{u}=0$ for all $\beta^{\prime}>\beta$, where $\tilde{u}$ denotes the extension of $u$ in $\mathcal{S}$ by 0$)$. Hence, $L_{\beta}$ is not of Fredholm type.

The second non trivial result we needed in $\S 4$ is a result of decomposition. Using a density process and residue formula, the following result can be proved in the same manner as Theorem 5.4.2 of ${ }^{12}$.

Theorem 5.2. Assume that $\kappa_{\sigma} \neq-1$. To simplify, assume $\kappa_{\sigma} \neq-3,-1 / 3$ as well. Consider $\beta^{1}$ and $\beta^{2}$ two real numbers such that $\beta^{1}<\beta^{2}$. Suppose no eigenvalue of $\mathscr{L}$ lies on the lines $\Re e\{\lambda\}=\beta^{1}, \Re e\{\lambda\}=\beta^{2}$, and that $\lambda_{1}, \cdots, \lambda_{N}$ are the eigenvalues of $\mathscr{L}$ in the strip $\beta^{1}<\Re e\{\lambda\}<\beta^{2}$. Let $u_{\beta^{2}}$ be an element of $\mathrm{W}_{\beta^{2}}^{1}(\mathcal{B})$ which satisfies $L_{\beta^{2}} u_{\beta^{2}} \in \mathrm{W}_{\beta^{1}}^{1}(\mathcal{B})^{*}$. Then, there holds the following representation

$$
u_{\beta^{2}}=u_{\beta^{1}}+\sum_{k=1}^{N} c_{k} \zeta e^{-\lambda_{k} z} \varphi_{k}
$$

where $u_{\beta^{1}}$ is an element of $\mathrm{W}_{\beta^{1}}^{1}(\mathcal{B}), \varphi_{k}$ is an eigenvector of $\mathscr{L}$ associated with the eigenvalue $\lambda_{k}, c_{k}$ is a constant (which depends on the choice of $\left.\varphi_{k}\right)$ and $\zeta \in \mathscr{C}^{\infty}\left(\mathbb{R}_{-}\right)$ is a cut-off function such that $\zeta(z)=1$ for $z \leq-2, \zeta(z)=0$ for $z>-1$.

Lemma 4.1. Let $\kappa_{\sigma} \in(-1,-1 / 3)$ and consider $\beta \in(0,2)$. The operator $L_{-\beta}$ : $\mathrm{W}_{-\beta}^{1}(\mathcal{B}) \rightarrow \mathrm{W}_{\beta}^{1}(\mathcal{B})^{*}$ is one-to-one, and there exists a constant $C>0$ such that

$$
\|v\|_{\mathrm{W}_{-\beta}^{1}(\mathcal{B})} \leq C\left\|L_{-\beta} v\right\|_{\mathrm{W}_{\beta}^{1}(\mathcal{B})^{*}}, \quad \forall v \in \mathrm{W}_{-\beta}^{1}(\mathcal{B}) .
$$

Proof. Take any $u \in \operatorname{ker} L_{-\beta}$, and define $\tilde{u}$ over $\mathcal{S} \stackrel{\text { def }}{=} \mathbb{R} \times(0, \pi)$ by $\tilde{u}(z, \theta)=$ $u(z, \theta)$ for $z \leq 0$, and $\tilde{u}(z, \theta)=-u(-z, \theta)$ for $z \geq 0$. Since $\left.u\right|_{\partial \mathcal{B}}=0$, we have $\tilde{u} \in \mathrm{H}_{\mathrm{loc}}^{1}(\mathcal{S})$. Besides, we have $(z, \theta) \mapsto e^{-\beta z} \tilde{u}(-z, \theta) \in \mathrm{H}^{1}(\mathcal{B})$, and in particular $(z, \theta) \mapsto e^{\beta z} \tilde{u}(-z, \theta) \in \mathrm{H}^{1}(\mathcal{B})$, so that $\tilde{u} \in \mathrm{W}_{-\beta}^{1}(\mathcal{S})$. Take any $v \in \mathrm{W}_{\beta}^{1}(\mathcal{S})$ and set $w(z, \theta)=v(z, \theta)-v(-z, \theta)$. We have $w(z, \theta) \in \mathrm{W}_{\beta}^{1}(\mathcal{B})$ since $\left.w\right|_{\partial \mathcal{B}}=0$. A straightforward calculus using the skew-symmetry of $\tilde{u}$ yields

$$
(\sigma \nabla \tilde{u}, \nabla v)_{\mathcal{S}}=(\sigma \nabla u, \nabla w)_{\mathcal{B}}=0 .
$$

As a consequence, we have $(\sigma \nabla \tilde{u}, \nabla v)_{\mathcal{S}}=0$ for all $v \in \mathrm{W}_{\beta}^{1}(\mathcal{S})$. Theorem 5.1 shows that the only $\tilde{u} \in \mathrm{W}_{-\beta}^{1}(\mathcal{S})$ satisfying such a property is $\tilde{u}=0$, so that $u=0$. The continuity of $L_{-\beta}^{-1}$ from range $L_{-\beta}$ to $\mathrm{W}_{-\beta}^{1}(\mathcal{B})$ is then a direct consequence of the open mapping theorem. 


\section{Appendix}

We begin this appendix by stating a result established in ${ }^{29}$ which is an extension of the well-known Peetre's lemma ${ }^{24}$ (see also ${ }^{32}$ ), that is particularly useful to prove that an operator is of Fredholm type.

Lemma 6.1. Let $\left(\mathrm{X},\|\|_{\mathrm{X}}\right),\left(\mathrm{Y},\|\|_{\mathrm{Y}}\right)$ and $\left(\mathrm{Z},\|\|_{\mathrm{Z}}\right)$ be three Banach spaces. Let $K: \mathrm{X} \rightarrow \mathrm{Z}$ be a linear compact map and $B: \mathrm{X} \rightarrow \mathrm{Y}$ be a continuous linear map. Suppose there exists $C>0$ such that

$$
\|x\|_{\mathrm{X}} \leq C\left(\|B x\|_{\mathrm{Y}}+\|K x\|_{\mathrm{Z}}\right), \quad \forall x \in \mathrm{X}
$$

Then $\operatorname{dim}(\operatorname{ker} B)<\infty$ and range $B$ is closed in $\mathrm{Y}$.

For a Banach space $\mathrm{X}$, let $\mathrm{X}^{*}$ refer to its topological dual and let $\langle,\rangle_{\mathrm{X}}$ be the duality pairing between $\mathrm{X}^{*}$ and $\mathrm{X}$. For an operator $L: \mathrm{X} \rightarrow \mathrm{X}^{*}$ that is self-adjoint, i.e. $\langle L u, v\rangle_{\mathrm{X}}=\langle L v, u\rangle_{\mathrm{X}} \forall u, v \in \mathrm{X}$, and such that range $L$ is closed, there exists a continuous isomorphism mapping $\operatorname{ker} L$ onto $(\text { coker } L)^{*}$, see Theorem 2.13 in ${ }^{17}$. In this situation, it is a consequence of previous lemma that any self-adjoint operator $L: \mathrm{X} \rightarrow \mathrm{X}^{*}$ is Fredholm as soon as it satisfies an a priori estimate such as (6.1).

Lemma 6.2. Consider the function $\varphi_{\mathrm{p}}(\theta)$ defined by Formula (4.9). Moreover, assume that $\sigma_{2} / \sigma_{1} \in(-1,-1 / 3)$. In this case, we have $\int_{0}^{\pi} \sigma(\theta)\left|\varphi_{\mathrm{p}}(\theta)\right|^{2} d \theta \neq 0$.

Proof. Recall that $\varphi_{\mathrm{p}}(\theta)$ defined by Formula (4.9) depends on a parameter $\eta_{\sigma}$, and that $\eta_{\sigma} \in(0,+\infty)$ whenever $\sigma_{2} / \sigma_{1} \in(-1,-1 / 3)$. For simplicity, $\eta_{\sigma}$ is denoted $\eta$ in this proof. Set

$$
I_{1}(\eta) \stackrel{\text { def }}{=} \int_{\pi / 4}^{\pi}\left|\varphi_{\mathrm{p}}\right|^{2} d \theta \quad \text { and } \quad I_{2}(\eta) \stackrel{\text { def }}{=} \int_{0}^{\pi / 4}\left|\varphi_{\mathrm{p}}\right|^{2} d \theta
$$

We are going to show that $\sigma_{1} I_{1}(\eta)+\sigma_{2} I_{2}(\eta)>0$ for all $\eta \in(0 ;+\infty)$. We have $\sigma_{1} I_{1}+\sigma_{2} I_{2}=\sigma_{1}\left(I_{1}+\kappa_{\sigma} I_{2}\right)>\sigma_{1}\left(I_{1}-I_{2}\right)$ so we study the function $\eta \mapsto I_{1}(\eta)-I_{2}(\eta)$. Explicit calculus using Formula (4.9) yields

$$
I_{1}(\eta)=\frac{\sinh 3 \eta \pi / 2-3 \eta \pi / 2}{2 \eta(\cosh 3 \eta \pi / 2-1)} \quad \text { and } \quad I_{2}(\eta)=\frac{\sinh \eta \pi / 2-\eta \pi / 2}{2 \eta(\cosh \eta \pi / 2-1)}
$$

Define $g(x) \stackrel{\text { def }}{=}(\sinh x-x) /(\cosh x-1)$. We have $2 \eta\left(I_{1}(\eta)-I_{2}(\eta)\right)=g(3 \eta \pi / 2)-$ $g(\eta \pi / 2)$, so it is sufficient to prove that $g$ is an increasing function on $(0,+\infty)$. One computes $g^{\prime}(x)=(2-2 \cosh x+x \sinh x) /(\cosh x-1)^{2}$. Define $g_{a}(x)=2-$ $2 \cosh x+x \sinh x$. One finds $g_{a}^{\prime}(x)=-\sinh x+x \cosh x$ and $g_{a}^{\prime \prime}(x)=x \sinh x$. One deduces, successively, $g_{a}^{\prime}>0$ and $g^{\prime}>0$. Thus $g$ is indeed an increasing function on $(0,+\infty)$. 


\section{References}

1. A.-S. Bonnet-Ben Dhia, L. Chesnel, and P. Ciarlet Jr. Optimality of $T$-coercivity for scalar interface problems between dielectrics and metamaterials. http://hal.archivesouvertes.fr/hal-00564312/, 2010.

2. A.-S. Bonnet-Ben Dhia, P. Ciarlet Jr., and C.M. Zwölf. A new compactness result for electromagnetic waves. Application to the transmission problem between dielectrics and metamaterials. Math. Models Meth. App. Sci., 18:1605-1631, 2008.

3. A.-S. Bonnet-Ben Dhia, P. Ciarlet Jr., and C.M. Zwölf. Time harmonic wave diffraction problems in materials with sign-shifting coefficients. J. Comput. Appl. Math, 234:1912-1919, 2010. Corrigendum J. Comput. Appl. Math., 234:2616, 2010.

4. A.-S. Bonnet-Ben Dhia, D. Dauge, and K. Ramdani. Analyse spectrale et singularités d'un problème de transmission non coercif. C. R. Acad. Sci. Paris, Ser. I, 328:717-720, 1999.

5. G. Cardone, S.A. Nazarov, and J. Taskinen. "Absorption" effect for elastic waves by the beak-shaped boundary irregularity. 54(3):146-150, 2009.

6. G. Cardone, S.A. Nazarov, and J. Taskinen. A criterion for the existence of the essential spectrum for beak-shaped elastic bodies. Journal de Mathématiques Pures et Appliquées, 92(6):628-650, 2009.

7. M. Costabel and E. Stephan. A direct boundary integral method for transmission problems. J. of Math. Anal. and Appl., 106:367-413, 1985.

8. M. Dauge and B. Texier. Problèmes de transmission non coercifs dans des polygones. Technical Report 97-27, Université de Rennes 1, IRMAR, Campus de Beaulieu, 35042 Rennes Cedex, France, 1997. http://hal.archivesouvertes.fr/docs/00/56/23/29/PDF/BenjaminT_arxiv.pdf.

9. N. Engheta. An idea for thin subwavelength cavity resonators using metamaterials with negative permittivity and permeability. Antennas and Wireless Propagation Letters, IEEE, 1:10-13, 2002.

10. P. Fernandes and M. Raffetto. Well posedness and finite element approximability of time-harmonic electromagnetic boundary value problems involving bianisotropic materials and metamaterials. Mathematical Models and Methods in Applied Sciences, 19(12):2299-2335, 2009.

11. V.A. Kondrat'ev. Boundary-value problems for elliptic equations in domains with conical or angular points. Trans. Moscow Math. Soc., 16:227-313, 1967.

12. V.A. Kozlov, V.G. Maz'ya, and J. Rossmann. Elliptic Boundary Value Problems in Domains with Point Singularities, volume 52 of Mathematical Surveys and Monographs. AMS, Providence, 1997.

13. V.A. Kozlov, V.G. Maz'ya, and J. Rossmann. Spectral problems associated with corner singularities of solutions to elliptic equations, volume 85 of Mathematical Surveys and Monographs. American Mathematical Society, Providence, RI, 2001.

14. VV Krylov. New type of vibration dampers utilising the effect of acoustic "black holes". Acta Acustica united with Acustica, 90(5):830-837, 2004.

15. J.-L. Lions and E. Magenes. Problèmes aux limites non homogènes et applications. Dunod, 1968.

16. D. Maystre and S. Enoch. Perfect lenses made with left-handed materials: Alice's mirror? JOSA A, 21(1):122-131, 2004.

17. W. McLean. Strongly elliptic systems and boundary integral equations. Cambridge University Press, Cambridge, 2000.

18. S.A. Nazarov and B.A. Plamenevsky. Elliptic problems in domains with piecewise smooth boundaries, volume 13 of Expositions in Mathematics. De Gruyter, Berlin, Allemagne, 1994. 
19. S.A. Nazarov and J. Taskinen. On the spectrum of the steklov problem in a domain with a peak. Vestnik St. Petersburg University: Mathematics, 41(1):45-52, 2008.

20. S.A. Nazarov and J. Taskinen. On essential and continuous spectra of the linearized water-wave problem in a finite pond. Mathematica Scandinavica, 2010.

21. S.A. Nazarov and J. Taskinen. Radiation conditions at the top of a rotational cusp in the theory of water-waves. ESAIM: Mathematical Modelling and Numerical Analysis, 45:947-979, 2011.

22. S. Nicaise and J. Venel. A posteriori error estimates for a finite element approximation of transmission problems with sign changing coefficients. Journal of Computational and Applied Mathematics, 235(14):4272-4282, 2011.

23. G. Oliveri and M. Raffetto. A warning about metamaterials for users of frequencydomain numerical simulators. Antennas and Propagation, IEEE Transactions on, 56(3):792-798, 2008.

24. J. Peetre. Another approach to elliptic boundary problems. Commun. Pure Appl. Math., 14:711-731, 1961.

25. J.B. Pendry. Negative refraction makes a perfect lens. Physical Review Letters, 85(18):3966-3969, 2000.

26. M. Raffetto. Ill-posed waveguide discontinuity problem involving metamaterials with impedance boundary conditions on the two ports. Science, Measurement $\mathscr{E}$ Technology, IET, 1(5):232-239, 2007.

27. K. Ramdani. Lignes supraconductrices: analyse mathématique et numérique. $\mathrm{PhD}$ thesis, Université Paris 6, 1999.

28. A.A. Sukhorukov, I.V. Shadrivov, and Y.S. Kivshar. Wave scattering by metamaterial wedges and interfaces. Int. J. Numer. Model., 19:105-117, 2006.

29. L. Tartar. Sur un lemme d'équivalence utilisé en analyse numérique. Calcolo, 24(2):129-140, 1987.

30. V.G. Veselago. The electrodynamics of substances with simultaneously negative values of $\varepsilon$ and $\mu$. Sov. Phys. Usp, 10:509-514, 1968.

31. H. Wallén, H. Kettunen, and A. Sihvola. Surface modes of negative-parameter interfaces and the importance of rounding sharp corners. Metamaterials, 2(2-3):113-121, 2008.

32. J. Wloka. Partial Differential Equations. Cambridge Univ. Press, 1987.

33. R.W. Ziolkowski and E. Heyman. Wave propagation in media having negative permittivity and permeability. Physical Review E, 64(5):056625, 2001.

34. C.M. Zwölf. Méthodes variationnelles pour la modélisation des problèmes de transmission d'onde électromagnétique entre diélectrique et méta-matériau. PhD thesis, Université Versailles - Saint-Quentin, 2008. 